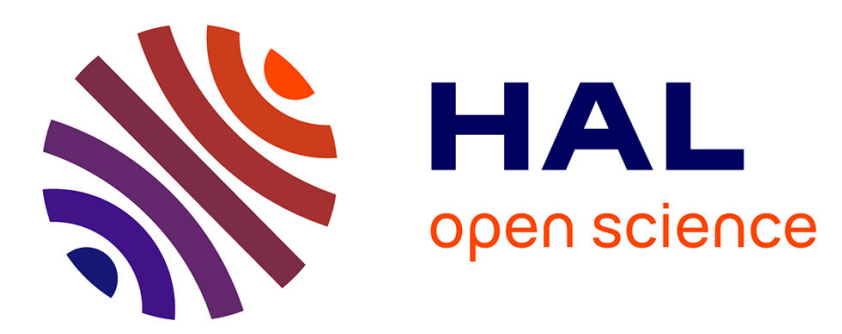

\title{
On the use of plasma synthetic jets for the control of jet flow and noise
}

\author{
M. Huet
}

\section{To cite this version:}

M. Huet. On the use of plasma synthetic jets for the control of jet flow and noise. AIAA, Jun 2014, ATLANTA, United States. hal-01069423

\section{HAL Id: hal-01069423 \\ https://hal-onera.archives-ouvertes.fr/hal-01069423}

Submitted on 29 Sep 2014

HAL is a multi-disciplinary open access archive for the deposit and dissemination of scientific research documents, whether they are published or not. The documents may come from teaching and research institutions in France or abroad, or from public or private research centers.
L'archive ouverte pluridisciplinaire HAL, est destinée au dépôt et à la diffusion de documents scientifiques de niveau recherche, publiés ou non, émanant des établissements d'enseignement et de recherche français ou étrangers, des laboratoires publics ou privés. 


\title{
On the use of Plasma Synthetic Jets for the control of jet flow and noise
}

\author{
M. Huet* \\ Onera - The French Aerospace Lab, F-92322, Châtillon, France
}

\begin{abstract}
The noise generated by an isothermal single stream jet at Mach number $M_{j}=0.9$ and diameter-based Reynolds number $R e_{D}=10^{6}$ is investigated numerically for an unforced configuration and a forced configuration with an active control device. This active flow forcing is performed with 12 Plasma Synthetic Jets (PSJs) located at the nozzle lip and acting on the axisymmetric mode of the shear layer of the main jet with a forcing Strouhal number $S t_{F}$ of 0.14. Simulated flow and noise results are validated with comparisons to available experiments performed for identical jet conditions. The noise radiation of the reference, unforced jet is globally well recovered numerically with a limited overestimation of the experimental pressure levels, below $3 \mathrm{~dB}$ for most of the observations angles. In the presence of the control, the simulated interaction between the fluid injected by the PSJs and the main flow reproduces very well the development of a large coherent vortex observed experimentally in the jet shear layer. The control is also found to favour the generation of turbulence. It translates for the acoustics to an increase of the broadband noise and of the integrated pressure levels of $1.5 \mathrm{~dB}$ in both experiments and simulations.
\end{abstract}

\section{Introduction}

Increasingly severe regulations concerning noise emission is a real challenge for any new aircraft. In particular, jet noise is a major contributor to overall aircraft noise during take-off operations and much attention remains focused on this subject. For subsonic jets, it is now universally admitted that noise comes from the flow mixing induced by the turbulence and that it is axially restricted to two potential core lengths. ${ }^{1,2}$ Acting on the turbulence that develops in the jet may therefore lead to a modification of its radiated noise. The systems used to act on the turbulence of the jet can be decomposed in two separate families, namely the passive and active control systems.

On the one hand, passive systems correspond to geometrical modifications of the nozzle, such as chevrons ${ }^{3-5}$ or tabs. ${ }^{6-8}$ Experimentally, Bridges \& Brown ${ }^{3}$ observed that the penetration of the chevrons strongly impacts jet centreline velocity decay and far-field noise. It lowers the radiated pressure at low frequencies but increases it at high frequencies. Concerning the aerodynamics, Alkislar et al. ${ }^{4}$ noticed increased turbulence levels in the initial shear layer. Acoustically, the authors evidenced similar noise modifications as Bridges \& Brown and explained the low frequency noise decrease by the breakdown of the large scale coherent structures at the jet exit whereas the increased mixing in the initial shear layer increases the high frequency part of the spectra. Despite their interest for noise reduction, such technologies lead to a diminution of the thrust during the whole cruise, which penalizes the performance of the engine.

On the other hand, active control systems present a major interest as they can be turned off when they are considered to be unnecessary. The thrust loss can therefore be limited to specific phases of the flight, such as the takeoff. Continuous air injection with microjets was shown to have a significant action on the jet. Depending on the mass flow rate and the number of microjets, the turbulence level can be either increased ${ }^{4,9}$ or reduced..$^{9,10}$ For the acoustics, it leads in all cases to a reduction in the low frequency range and to an increase it for large frequencies, for all observation angles. ${ }^{11}$ The OASPL reduction varies between $0.5 \mathrm{~dB}$ and $2 \mathrm{~dB}$, depending on the configuration of the microjets. A drawback of such continuous microjets is that the air they blow has to be taken from the engine, typically from the bypass flow, which penalizes the thrust. To reduce or suppress the mass flow to be taken from the engine, one may want to use pulsed actuators.

*Research scientist, CFD and aeroacoustics department, Maxime.Huet@onera.fr 
Ragaller, ${ }^{12}$ for instance, demonstrated the capacity of achieving with a reduced mass flow pulsed control a noise decrease close to that obtained with continuous water injection.

For the control of subsonic jets with pulsed actuators, one can cite the Localized Arc Filament Plasma Actuators (LAFPAs) that have demonstrated to be efficient in flow and noise control. ${ }^{13-17}$ LAFPAs consist in a pair of pin electrodes and generate electric discharge plasmas at a driven frequency varying from 0 to $200 \mathrm{kHz}$. The authors especially observed a broadband noise increase for low forcing frequency excitation, below $S t_{F}=1$, and a reduction for higher frequencies. For these actuators, it is the pure energy injected in the jet that perturbs the flow. The action of this control on the jet thus differs from that of continuous microjets, where the injected momentum is thought to act as the main element for flow control. Using a technology similar to LAFPAs, Onera has developed Plasma Synthetic Jets (PSJs), where the electrodes are embedded in a ceramic cavity with an exit orifice. ${ }^{18,19}$ The energy deposit inside the cavity increases the local temperature and pressure which generates a jet outside the cavity. This way, PSJs act on the mean flow in a very similar way as continuous microjets.

The current study is performed in the frame of the European project ORINOCO, dedicated to the control of engine noise with plasma actuators. To this end, unsteady simulations are used in the present work to reproduce numerically the flow development and noise radiation of an isothermal jet with an exhaust Mach number of 0.9 controlled with the PSJs developed at Onera. ${ }^{20-22}$ This jet configuration has been studied experimentally in Lyon by the LMFA - École Centrale de Lyon and by Onera during the project. Two jet flow simulations and associated noise radiation are performed to demonstrate the capacity of the numerical tools to reproduce the flow development and its interaction with the PSJs. The first simulation corresponds to the baseline configuration, without control. It is used in the following as a reference simulation to exhibit the flow modifications caused by the use of the actuators. In the second simulation, the flow is controlled by 12 PSJs located at the nozzle lip. Simulated flow data are compared to experimental results obtained at the the Réchauffeur 113 facility of Onera, in Toulouse, for the configuration without PSJs. Acoustic measurements without and with PSJs, performed in the anechoic facility of the Centre Acoustique, LMFA - École Centrale de Lyon, are used as a reference for the validation of the simulated far-field noise.

The document is organized as follows. The numerical procedure is detailed in section II. This section especially contains the details of the jet investigated and the numerical methods used in the simulations. Details on the method used to seed turbulence in the nozzle boundary layers are then presented in section III. The characteristics of the PSJs and their numerical modelling are presented in section IV. In section V, a detailed flow analysis is performed. The action of the PSJs on the flow development is especially highlighted. The noise radiated to the far field in the two simulations is then presented in section VI and compared to reference experimental data. To end, conclusions and perspectives are finally drawn in section VII.

\section{Numerical Procedure}

\section{II.A. Jet configuration}

The jet considered in the present study is an isothermal air jet with a nozzle of diameter $D=2 r_{0}=50 \mathrm{~mm}$, a Mach number $M_{j}=U_{j} / c_{j}=0.9$ and a diameter-based Reynolds number $R e_{D}=U_{j} D / \nu=10^{6}$, where $U_{j}$ is the jet exhaust velocity, $c_{j}$ the speed of sound in the jet and $\nu$ the kinematic viscosity. A sketch of the nozzle is visible in Fig. 1. It is the exact geometry that has been used for the tests in the project. The internal lines of the nozzle are similar to those used in the projects JEAN ${ }^{23,24}$ and OSCAR. ${ }^{25}$ In order to integrate the PSJs to the geometry for the experiments, the nozzle lip has been increased for the present geometry compared to the JEAN nozzle and has a thickness of $15 \mathrm{~mm}$.

The jet conditions are identical for the two simulations. They correspond to a static configuration and are reported in Table 1 , where $p_{0}$ and $T_{0}$ stand for the mean pressure and mean temperature in the medium at rest and $p_{i}$ and $T_{i}$ to the stagnation pressure and stagnation temperature, respectively.

\begin{tabular}{cccc}
\hline$p_{0}$ & $T_{0}$ & $p_{i} / p_{0}$ & $T_{i} / T_{0}$ \\
\hline \hline $100,000 \mathrm{~Pa}$ & $280 \mathrm{~K}$ & 1.691303 & 1.162 \\
\hline
\end{tabular}

Table 1. Inlet and outlet flow conditions used in the simulations.

Simulations are run for a static configuration, without external flow. For numerical stability purpose, however, an external flow $U_{\infty}=5 \mathrm{~m} / \mathrm{s}$ is imposed in the simulations. It corresponds to a usual approach for 


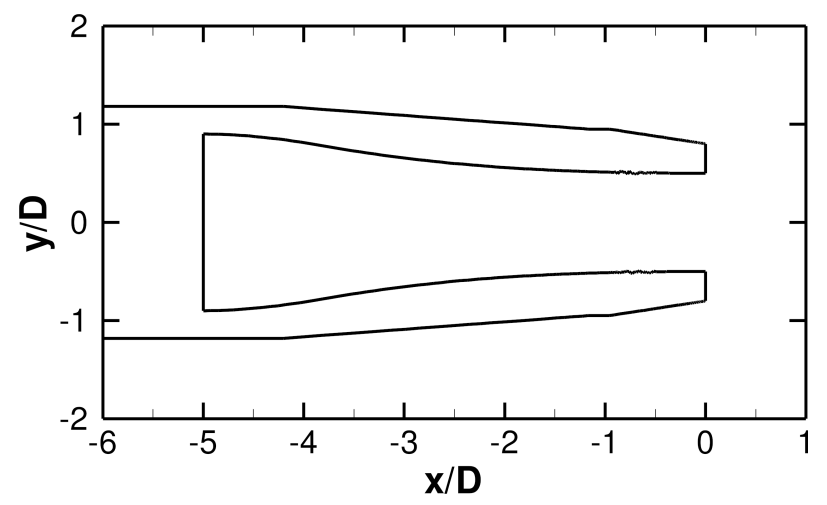

Figure 1. Nozzle shape

the simulations performed at Onera ${ }^{25,26}$ and the low velocity of the external flow is expected not to modify the flow characteristics and noise radiation.

\section{II.B. Flow resolution}

The numerical simulations used to compute the noise radiated by the jet rely on a hybrid approach, that combines a flow simulation to determine the noise sources and near field fluctuations with an integral formulation to radiate the noise to the observer locations. With this approach, the expensive flow simulation is restricted to the jet development area, where turbulence and nonlinear phenomena occur, while the acoustic propagation in a uniform medium is achieved with an affordable radiation formulation. This approach has been successfully used at Onera during the last decade, see for instance Huet et al. ${ }^{25}$ and Huet. ${ }^{26}$

\section{II.B.1. Numerical methods}

Aerodynamic simulations are performed using the flow solver CEDRE developed at Onera. CEDRE is a multi-physics, reactive solver used by researchers and aeronautical industries for engine conception and optimisation, such as combustion, ${ }^{27,28}$ turbine blade cooling ${ }^{29}$ and jet noise ${ }^{25,26,30}$ for instance. The resolution of the Navier-Stokes equations is made for the conservative variables and is based on a finite volume formulation using a second order upwind space discretization scheme for generalised polyhedral computational grids. ${ }^{31}$ Time integration is currently made with a first order implicit time scheme. Unsteady flow fields are computed using LES with a Smagorinsky subgrid-scale model. ${ }^{32}$

The boundary conditions used for the simulations are the following. At the outflow boundary, downstream of the jet flow, the static pressure is imposed. For upstream and lateral boundaries, static temperature and velocity are imposed. Inside the nozzle, flow injection is made through uniform stagnation pressure and temperature values. The internal boundary layer profile is thus not imposed and is expected to develop freely along the nozzle wall. Its thickness is expected to depend on the grid resolution and numerical schemes precision. Details of the near-wall region grid sizing are given in section II.B.2.

Simulations are performed with a time step $\Delta t=3 \times 10^{-7} \mathrm{~s}$ which ensures a CFL criterion below 1 in almost all the numerical domain. After a transient period of $24 \mathrm{~ms}(80,000$ iterations) required to let the jet flow develop, the simulations are run for 234,000 iterations to perform mean flow averaging and far-field noise radiation. It corresponds to a physical time of $70.20 \mathrm{~ms}$ or to a non-physical time of 420 convective time units $D / U_{j}$. Previous simulations of a similar case ${ }^{25}$ have demonstrated that this duration is sufficient to provide a statistical convergence of the flow fields and far-field pressure spectra.

\section{II.B.2. Grid definition}

The grid used for the present simulations is identical for the two configurations computed and follows the methodology used for the simulations of a hot single stream nozzle with a diameter of $80 \mathrm{~mm}$, where encouraging comparisons with the experiments have been made. ${ }^{26}$ The nozzle being axisymmetric and with a simple shape, it has been possible to construct a grid composed of structured hexahedra solely to bring under control the flow development both in the boundary layers and the jet plume. This approach differs 
with the one usually performed at Onera, ${ }^{25,33,34}$ where the grid combines a non-structured grid to easily discretize the complex nozzle geometry (especially in the presence of a plug, chevrons, a pylon and/or a wing) with a structured patch in the jet plume to locally increase the flow accuracy in the region of turbulence and sound production. The grid is composed of $40 \times 10^{6}$ elements.

The computational domain is a cylinder of radius $80 D$ that extends axially from $-20 D$ to $100 D$, with the nozzle exit located at $x=0$. This domain includes a refined zone composed of the nozzle and the jet plume, where the noise sources are located. This latter part corresponds to a truncated cone starting at the nozzle exit with an axial extent of $25 D$ and whose radius is $2 D$ at $x=0$ and $4 D$ at $x / D=25$. Outside this refined zone the grid is progressively stretched to damp the flow structures and acoustic waves before they reach the domain boundaries.

The refined zone corresponds to the jet plume and the near field. In the jet plume, the grid density is driven by the necessity to accurately reproduce de turbulent structures that mainly grow in the shear layer. The size of the elements is essentially conditioned by the resolution at nozzle exit, discussed later in this section. These structures produce acoustic waves that propagate in the near field to the storage surfaces (see section II.C). It is chosen to accurately propagate the acoustic waves to a Strouhal number $S t=f D / U_{j}$ of 1.2 where $f$ is the wave frequency. It corresponds in the present case to a maximum frequency $f_{\max }=7$ $\mathrm{kHz}$. This choice ensures that most of the acoustic energy is captured in the simulations. ${ }^{25}$

Experimentally, the importance of the turbulence levels in the boundary layer on the jet development and noise radiation has been evidenced since the 1980s. ${ }^{35-37}$ More recently, numerical results have confirmed the necessity to reproduce in the best possible way the turbulent behaviour of the boundary layer inside the nozzle to accurately simulate the jet flow and its noise. ${ }^{26,38,39}$ To this end, special attention is focused on the discretization of the nozzle boundary-layer. The boundary-layer turbulence will be discussed in section III.

For jets with a Reynolds number $R e_{D} \sim 10^{6}$, Zaman ${ }^{35,36}$ experimentally observed a momentum thickness of the nozzle internal boundary layer $\delta_{\theta} / D \sim 10^{-3}$. Because of computational restrictions, a larger value is usually used in the simulations, as done for instance by Bodony \& Lele, ${ }^{40}$ Bogey et al. ${ }^{41}$ and Bodard et $a l .{ }^{30}$ For the simulation of a $R e_{D}=500,000$ jet with a laminar boundary layer, Bogey et al. ${ }^{38}$ imposed a shear-layer thickness $\delta / D=0.064$ corresponding to a momentum thickness $\delta_{\theta} / D=0.008$. As done in a previous study at Onera, ${ }^{26}$ in the present simulations a shear-layer thickness $\delta_{\theta} / D$ of 0.05 is chosen to be discretized with 20 evenly spaced cells, which corresponds to $\Delta r / D=0.0025$ at the wall. The configurations to compute being static, no focus is given on the resolution of the external boundary layer. An illustration of the grid at the vicinity of the nozzle exhaust is visible in Fig. 2.

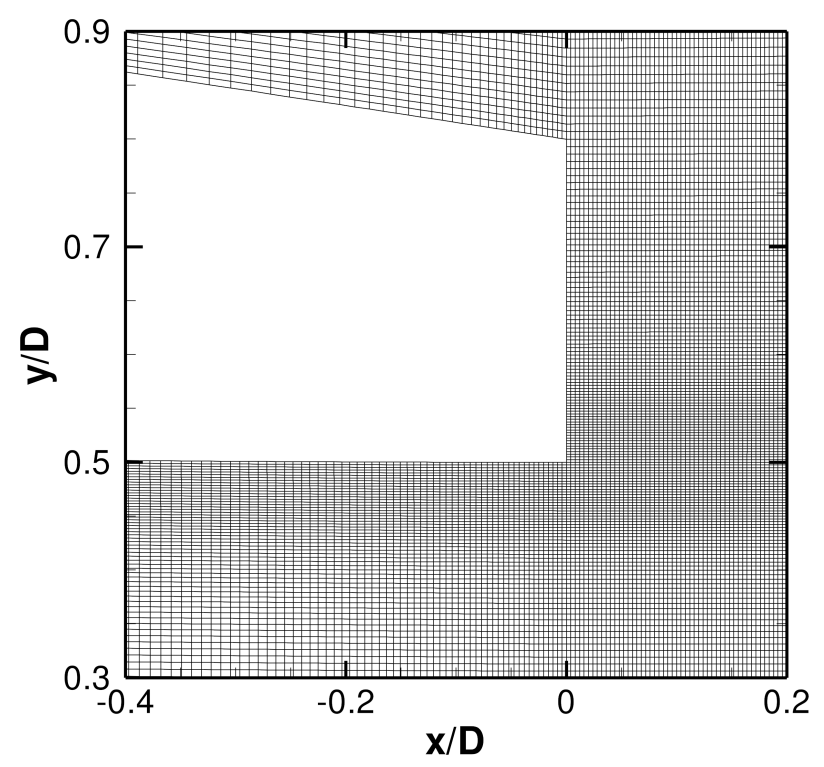

Figure 2. Detail of the numerical grid at nozzle exit.

Outside of the boundary layer, the flow gradients being lower the grid is radially stretched to reduce the total number of points. The radial evolution of the radial grid size at the nozzle exit $(x=0)$ is reproduced in Fig. 3 (a). The shear layer is estimated to be twice as large as the boundary layer at nozzle exit and is 
discretized by 40 cells whereas stretchings of $3 \%$ and $2 \%$ are applied towards the jet axis and outside the jet, respectively. This stretching is especially tuned to accurately capture the flow aerodynamics in the jet plume and the acoustic waves propagating in the near field to the surfaces used for the acoustic radiation.

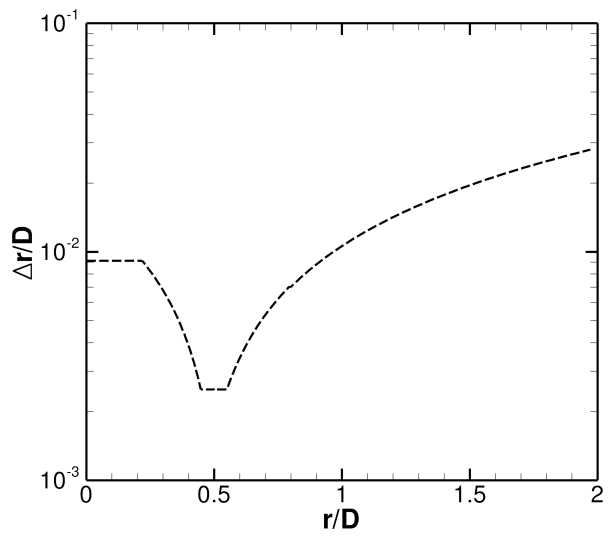

(a) radial evolution at $x / D=0$

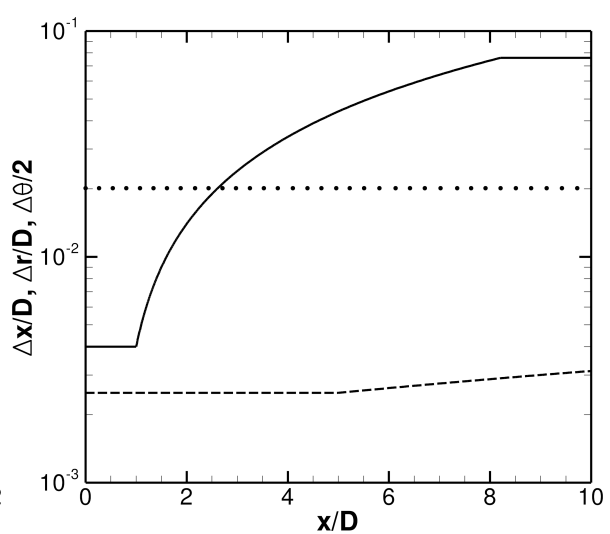

(b) axial evolution at $r=r_{0}$

Figure 3. Grid discretization in axial and radial directions. $-\Delta x / D ;---\Delta r / D ; \bullet r_{0} \Delta \theta / D$.

The grid discretization in the axial, radial and orthoradial directions along the jet shear layer is reproduced in Fig. 3 (b). Axially, the grid spacing is fixed to $\Delta x / D=0.004$ at nozzle exit. It is kept constant in the first diameter downstream of the exhaust and then stretched with a ratio of $1 \%$ to its maximum value of $\Delta x / D=0.076$, reached at $x / D=8.3$. This maximum cell size is then conserved up to the end of the refined zone, at $x / D=25$. The radial discretization is fixed at the nozzle exit by the resolution of the boundary layer in the nozzle. This grid size is conserved during the 5 first diameters of the jet and then slowly increased. In the azimuthal direction the grid is composed of 156 cells, which corresponds to a resolution $r_{0} \Delta \theta / D=0.0197$ at the nozzle lip. To end with the grid description, a O-type treatment is applied on the jet axis to ensure that hexahedral cells have an homogeneous size in the core region.

\section{II.C. Noise radiation}

The noise radiation is performed using the Ffowcs Williams \& Hawkings (FW-H) porous surface formulation ${ }^{42}$ available in the code KIM developed at Onera. ${ }^{43}$ This formulation makes it possible to compute time pressure histories at any observer location by integration of the flow fields on a control surface surrounding the jet and containing all the noise sources. It is preferred to the Kirchhoff method that was shown by Rahier et $a l .{ }^{43}$ to be more exposed to the generation of spurious noise, especially for hot jets.

The microphones are located $40 \mathrm{D}(2 \mathrm{~m})$ from the nozzle exit, as done in the experiments performed in the anechoic facility of the Centre Acoustique, LMFA - École Centrale de Lyon. To ensure the stability of the simulated pressure levels with respect to the azimuthal position of the microphones, simulated pressure spectra and integrated levels are averaged azimuthally on 36 microphones for each observer location. It has indeed been observed that radiated noise may vary up to $2 \mathrm{~dB}$ along the azimuthal position despite the axisymmetry of the configuration because of the short-time duration of the simulated signals. ${ }^{44}$

Following the results of Rahier et al., the control surface axially extends in the downstream direction up to the end of the refined zone, at $x / D=25$. In the upstream direction, the surface extends to $x / D=-2$. This location has been updated compared to the recent simulations ${ }^{25,26}$ where the surface used to start at $x / D=0$ and where radiated noise was observed to collapse at aft angles, above $120^{\circ}$. The surface is kept open at both extremities to avoid the contamination of the pressure signals by spurious noise generated by the crossing of the surface by turbulent spots.

Radially, the surface must on the first hand surround all the noise sources and hence must be located outside of the sound producing region. On the other hand, to limit the numerical dissipation the surface must also be located close enough from the jet plume. The correct radial position of the surface has therefore to be ensured after the aerodynamic simulation is complete. To validate its radial position, in the present study the radiation has been performed for 3 distinct surfaces. It has been observed that radiated pressure does not depend on the position of the surface and results are therefore presented for one reference surface only. Such comparisons moreover evidenced that the numerical dissipation is negligible for frequencies below 


\section{Turbulence triggering}

According to the experimental results of Zaman ${ }^{35-37}$ and more recent numerical observations, ${ }^{26,38,39}$ jet flow development and noise radiation vary with the turbulence level in the boundary layer at nozzle exit. For low turbulence configurations especially, the flow development is initially laminar with organized vortex shedding and pairings that lead to an additional noise source particularly important in the medium frequency range and for angles close to $90^{\circ}$.

Previous experiments performed by Castelain ${ }^{45}$ in the anechoic facility of the Centre Acoustique, LMFA - École Centrale de Lyon - for a similar nozzle demonstrated the initially turbulent development of the jet. Despite the absence of precise turbulence measurements at nozzle exit, one can however expect the turbulence to be at least of a few percent of the jet exhaust velocity.

Numerically, it has been demonstrated that the flow development itself in the nozzle is not sufficient to develop significant turbulence. ${ }^{26}$ More quantitatively, the axial velocity fluctuation $u_{x \mathrm{rms}} / U_{j}$ remains below $0.2 \%$ and a triggering inside the nozzle is necessary to generate high turbulence levels. In the present simulations, the choice has been made to use a geometrical disturbance inside the nozzle to generate some turbulence. The disturbance being constant in time, it is expected to generate negligible spurious noise. An illustration of the grid modification inside the nozzle to trigger the turbulence is illustrated in Fig. 4. The geometrical disturbance is random in both axial and azimuthal directions.

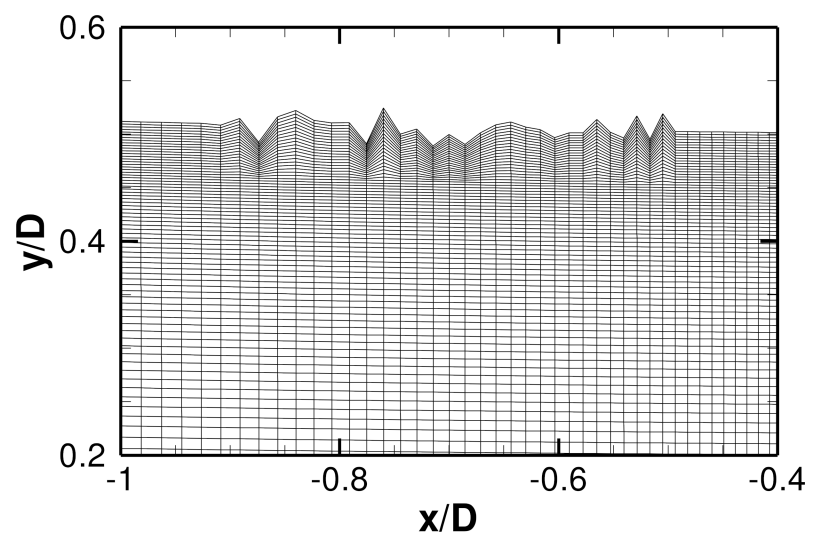

Figure 4. Nozzle geometry modifications performed for turbulence triggering.

The parameters of the triggering have been determined empirically with a trial and error approach. Multiple short-time duration simulations have been performed to evaluate the turbulence at nozzle exit and flow development for various positions and amplitudes of the geometrical disturbance. Finally, an axial extent from $x / D=-0.9$ to $x / D=-0.5$ and an amplitude of $\pm 0.02 D(1 \mathrm{~mm})$ have been retained.

\section{III.A. Boundary layers mean flow and turbulence development}

Figure 5 (a) illustrates the mean axial velocity simulated at nozzle exit without and with triggering. The boundary layer thickness, defined as the distance from the wall where the velocity is $99 \%$ of $U_{j}$, is largely increased by the geometrical disturbances. Blasius boundary layer profiles with identical boundary layer thicknesses (see Bogey et al. ${ }^{38}$ ) are also represented in the figure. Simulated and Blasius profiles clearly differ for both simulations, indicating that the simulated boundary layers are not laminar at nozzle exit. The simulated velocity profile is sharper than the Blasius one for the reference nozzle, whereas the opposite is observed with the triggered one.

Displacement thicknesses, momentum thicknesses and shape factors of the simulated boundary layers are reproduced in Table 2 for the two nozzles. The definition of these values is the following:

$$
\delta_{\star}=\int_{0}^{\infty}\left(1-\frac{u_{x}(r)}{U_{j}}\right) d r \quad \text { displacement thickness }
$$




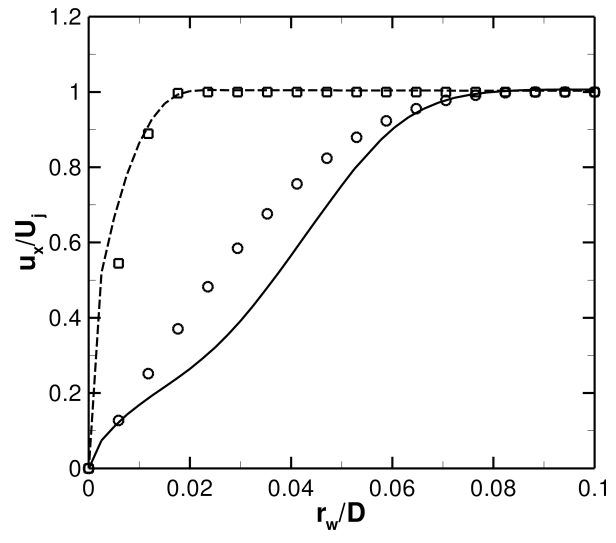

(a) mean axial velocity

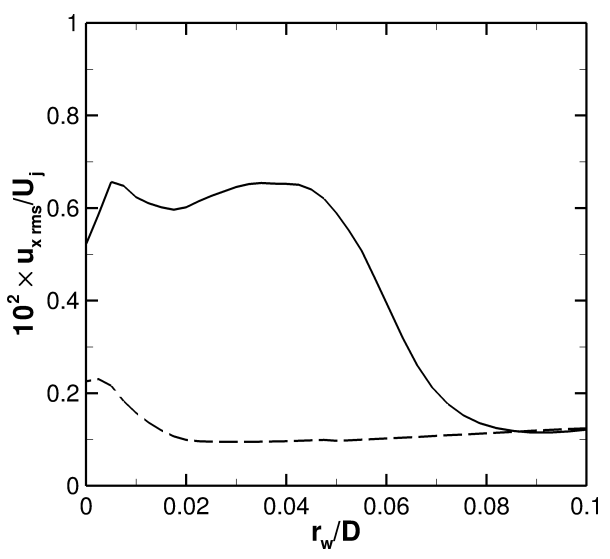

(b) turbulent axial velocity

Figure 5. Radial profile of (a) mean and (b) turbulent axial velocity at nozzle exit. - - - reference nozzle; - triggered nozzle. Symbols: Blasius profiles.

$$
\begin{array}{lll}
\delta_{\theta}=\int_{0}^{\infty} \frac{u_{x}(r)}{U_{j}}\left(1-\frac{u_{x}(r)}{U_{j}}\right) d r & \text { momentum thickness } \\
H=\frac{\delta_{\star}}{\delta_{\theta}} & \text { shape factor }
\end{array}
$$

For both simulations, the shape factor differs from the value of a laminar boundary layer $(H=2.59)$. In accordance with the results of Fig. 5 (a), $H<2.59$ for the reference nozzle and $H>2.59$ for the triggered one. The geometrical disturbance therefore strongly disturbs the boundary layer development. The large value of the shape factor, higher than for laminar boundary layer, may indicate that the distance between the disturbance and the nozzle exit is not sufficient for the flow to recover a state at equilibrium. This assumption is confirmed by the azimuthal variations observed at nozzle exit for the mean axial velocity, not reproduced here.

\begin{tabular}{ccc}
\hline & reference nozzle & triggered nozzle \\
\hline \hline Boundary layer thickness $\delta / D$ & 0.02 & 0.09 \\
Displacement thickness $\delta_{\star} / D$ & $6.05 \times 10^{-3}$ & $3.49 \times 10^{-2}$ \\
Momentum thickness $\delta_{\theta} / D$ & $3.75 \times 10^{-3}$ & $1.16 \times 10^{-2}$ \\
Shape factor $H$ & 1.61 & 3.01 \\
\hline
\end{tabular}

Table 2. Characteristic dimensions of the simulated boundary layers at nozzle exit.

To end with the profiles at nozzle exit, the turbulent axial velocity profile is reproduced in Fig. 5 (b). As observed in some previous simulations, ${ }^{26}$ the turbulence does not exceed $0.2 \%$ of the jet exhaust velocity for the reference nozzle. It increases above $0.6 \%$ in the presence of the triggering. This value remains low compared to the fluctuations of several percents expected in the experiments for a jet with a Reynolds number of $10^{6}, 35,36$ it lies however in the range of the maximum fluctuation that can be generated with the current method and parameters used. A larger amplitude of the geometrical disturbances or a more important axial extent of the triggering surface seems to be requested to reach higher turbulence levels; these latter modifications may however increase the impact on the mean flow fields. Another solution would be to move the triggering zone more upstream of the nozzle and let the turbulence develop on a longer axial distance. Indeed, as illustrated in Fig. 6 the peak turbulent axial velocity grows very fast between the forcing zone and the nozzle exit and increasing the axial distance between the forcing and the nozzle exhaust might lead to higher turbulence values at the nozzle exit. Increasing this distance would also help the mean flow to recover a canonical boundary layer profile. Such a procedure however requires a grid sufficiently dense to ensure an accurate convection of the fine scale generated turbulence to the nozzle exit and is not used in the present simulations. 


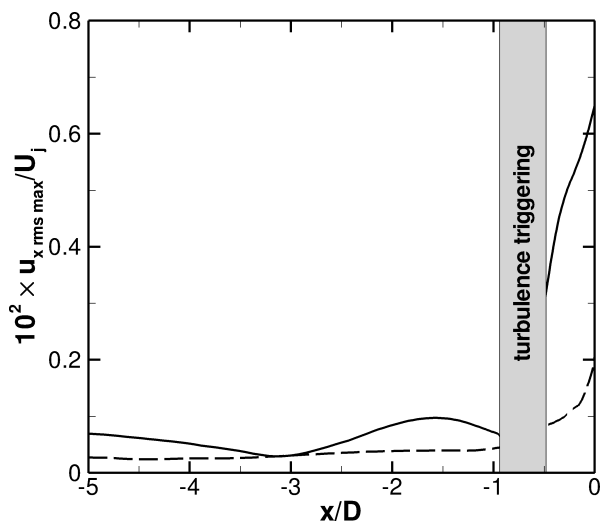

Figure 6. Axial evolution of the peak turbulent axial velocity inside the nozzle. --- reference nozzle; - triggered nozzle.

\section{III.B. Jet flow development}

The influence of the turbulence level at nozzle exit is now highlighted by comparing the simulated flows just downstream of the nozzle. Figure 7 represents instantaneous vorticity snapshots $\vec{\omega}=\vec{\nabla} \times \vec{u}$ for the two simulations. For the reference nozzle, Fig. 7 (a), the generation of structured vortices is visible in the shear layer just downstream of the nozzle exit, for $x / D \sim 0.2$. A first step of pairing is visible for $x / D \sim 0.5$ and may be followed downstream by more pairings. This flow development is characteristic of initially laminar shear layers and is coherent with the very low turbulence level observed in the shear layer at nozzle exit. For the triggered nozzle, Fig. 7 (b), the vortex shedding seems also to be less organized and the presence of pairings is less obvious than for the reference nozzle. The shear layer thickness seems also to be reduced compared to the reference nozzle for $x / D>2$. The too fast growth of the shear layer, leading to an underestimation of the potential core length compared to experiments, is often associated with the initially too laminar numerical jet development. These observations therefore tend to indicate that the turbulence seeding favours the turbulent transition of the jet.

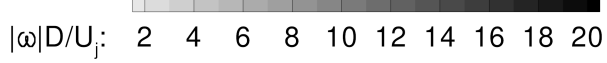

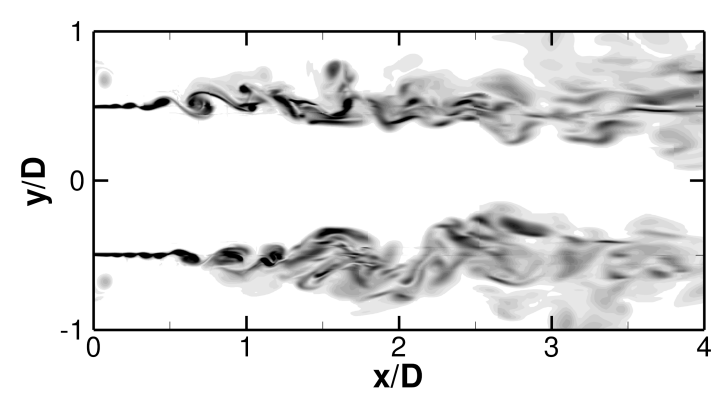

(a) reference nozzle

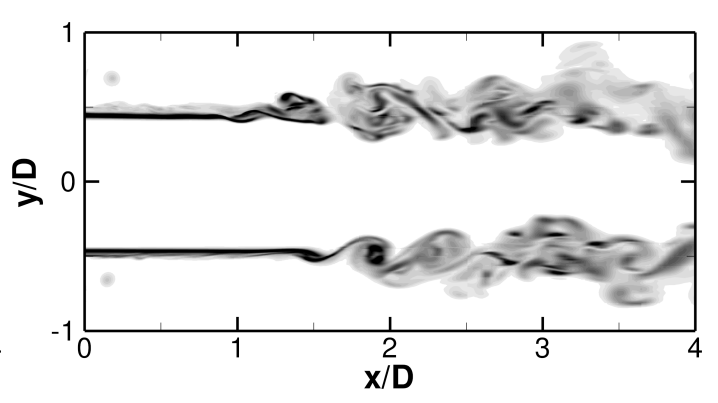

(b) triggered nozzle

Figure 7. Vorticity snapshots at nozzle exit.

To complete the analysis on the vorticity development, the evolution of the peak turbulent axial velocity along the axial direction is reported in Fig. 8. The turbulence level starts from a very low value at nozzle exit and grows in the first diameters where it reaches its maximum value, before decreasing downstream. This evolution is characteristic of initially laminar shear layers and has been observed in previous simulations with low inlet turbulence levels. ${ }^{26,38}$ It confirms the previous observations that the turbulence seeding is not sufficient to provide an initially turbulent flow at the nozzle exit. The results nevertheless illustrate the improvement of the turbulent evolution of the jet with the geometrical disturbances. As a consequence, jets simulations are performed in the following with the turbulence triggering method. 


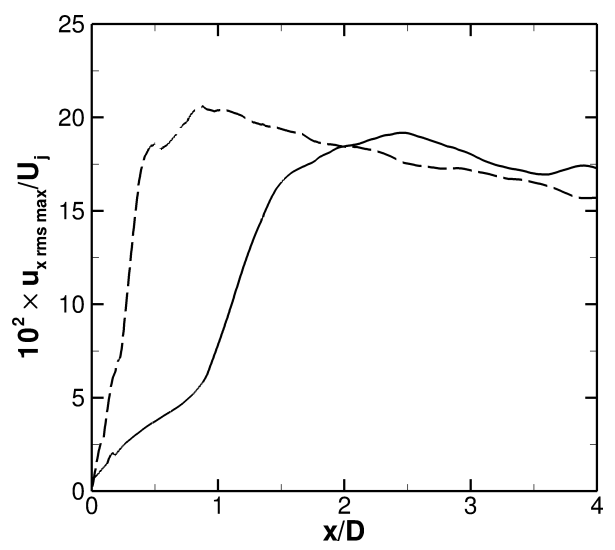

Figure 8. Axial evolution of the simulated peak turbulent axial velocity. - - - reference nozzle; — triggered nozzle.

\section{PSJs characteristics and numerical modelling}

Experimentally, the flow is controlled by 12 PSJs located in the nozzle lip, at a radial distance $r / D=0.6$ from the jet axis. PSJs are actuators composed of a ceramic cavity with an exit orifice of diameter $D_{P S J}=1$ $\mathrm{mm}$ and 2 electrodes. Each PSJ is supplied by high voltage pulses leading to arc discharges, which correspond to an energy deposit inside the cavity. The local increase of temperature and pressure generates a jet blowing outside the cavity. The fluid expulsion is followed by a natural recovery of the cavity that returns to ambient conditions. The jet is labelled synthetic because the mass flow is nil for each cycle.

The PSJs are equally spaced in azimuth with a separation angle of $30^{\circ}$. They are oriented towards the main jet with an angle of $45^{\circ}$ and have a yaw angle of $0^{\circ}$. Their frequency and phase have been chosen based on experimental results performed in the anechoic facility of the Centre Acoustique, LMFA - École Centrale de Lyon. They correspond to a configuration where the far-field noise modifications were found to be high. All microjets are run in phase (mode $m=0$ ) with a forcing frequency $f_{F}=820 \mathrm{~Hz}\left(S t_{F}=0.14\right)$. With this configuration of PSJs, a broadband noise increase of about $2 \mathrm{~dB}$ is observed experimentally.

The microjets modelling follows the methodology used previously for the simulations of continuous microjets, ${ }^{25}$ where a satisfactory agreement was found with the experiments. With this approach, microjets nozzles are not discretized in the computational grid; PSJs are modelled with source terms in the equations of mass, momentum and energy for specific cells corresponding to the locations of the actuators and are aimed to reproduce PSJs reference velocity and temperature. Using this modelling, the total number of cells is limited compared to a fine description of the PSJs and simulations can be run with a reasonable time step. In the present case, PSJs are modelled with a few cells only. One cannot expect to finely reproduce the turbulence generated by the actuators. The main objective of the simulations is to reproduce the injection of momentum that interacts with the main jet and which is thought to be of primary importance.

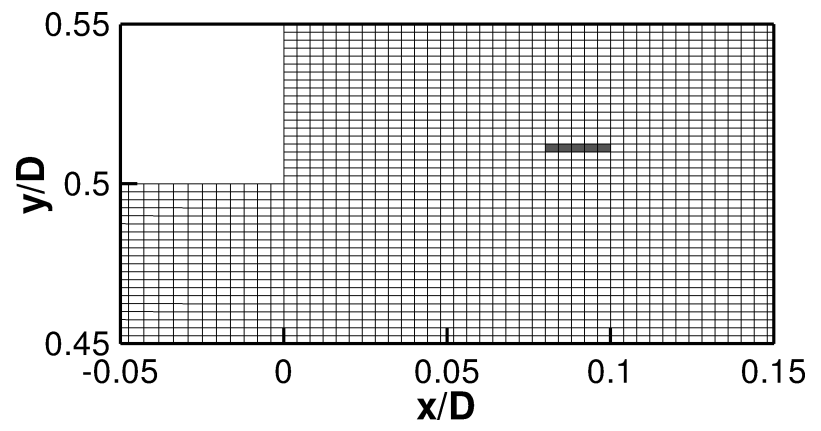

Figure 9. Illustration of the cells used to model the PSJ (in grey).

An illustration of the cells used to model one PSJ is given in Fig. 9. Each PSJ is modelled with 5 cells in the jet direction and 1 cell in the radial and azimuthal directions. The equivalent diameter of the actuators is estimated using the flow velocity and its orientation. Its value is $1.02 \mathrm{~mm}$, in agreement with the experimental diameter. 
Experimentally, PSJs are located at the nozzle lip. Measurements have indicated that the diffusion of the pulsed jets is weak and their velocity during the interaction with the main jet is expected to be similar to the flow velocity at PSJs exhaust. Numerically, the flow diffusion between the PSJs exhaust and the shear layer is more important because of the limited grid resolution. To limit this diffusion and reproduce the high microjet velocity during the interaction between the PSJ and the main flow, actuators are moved in the simulations towards the shear layer. Their location is chosen so that the interaction between the microjets and main jet still occurs at the same physical position.

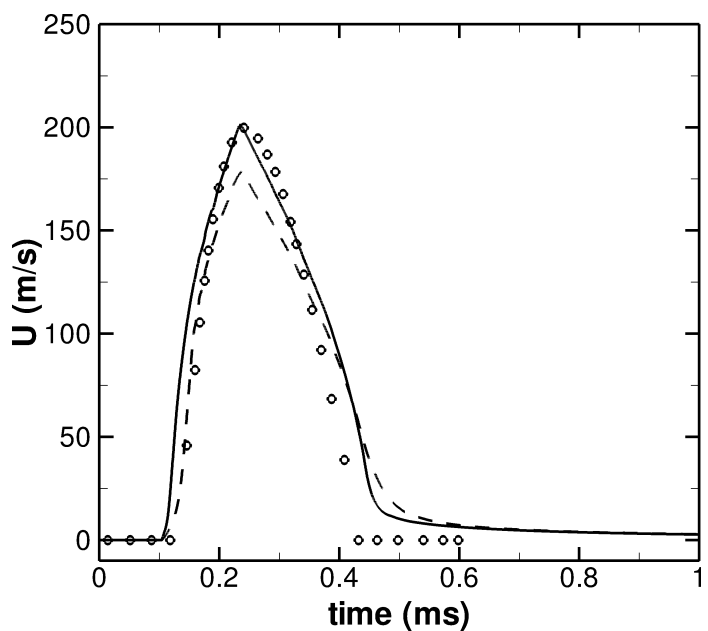

Figure 10. Velocity signal generated by the PSJ. $\circ$ experimental data at PSJ exit. - simulation at PSJ exit; - - simulation at main jet shear-layer location.

In the simulation, the temporal evolution of the source terms is tuned to fit the experimental velocity signal measured at PSJ exhaust. Experimental and numerical signals are reproduced in Fig. 10. In this figure, the numerical velocity signal at the radial position $r / D=0.5$ (main jet shear layer) is also plotted. Its high value illustrates the limited diffusion of the PSJ flow before its interaction with the main jet. The maximum temperature increase is set to $100 \mathrm{~K}$. This value has been determined numerically with the help of detailed numerical simulations of the PSJs. The velocity field generated by the PSJs in the present simulations is illustrated in Fig. 11.

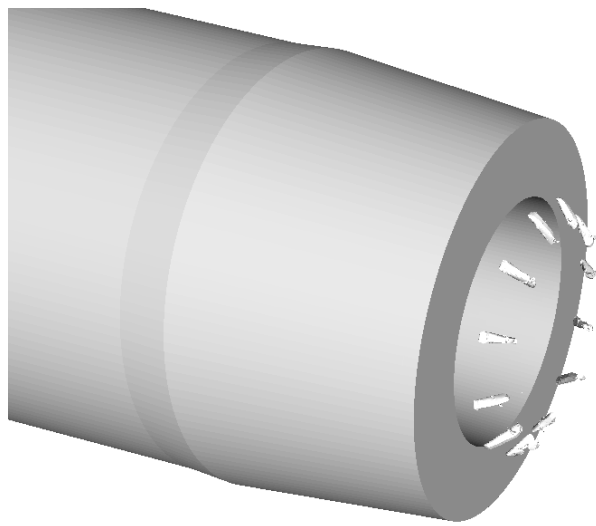

Figure 11. Velocity isosurface of the fluid injected by the PSJs.

To end, one must note that, in Fig. 10, simulated PSJ velocity is always positive. The simulated mass flow rate over each cycle is thus not nil, which differs from the experimental actuators. The negative flow velocity induced by the cavity filling with air is small and is not visible in the experimental signal. It is expected to have a negligible influence on the flow development and it is therefore neglected in the simulations. 


\section{Flow analysis}

In this section, the flow results of the baseline configuration are presented and validated by comparisons with experiments performed at Onera. The action of the PSJs on the jet flow development is also discussed based on the results of the numerical simulations.

The flow inside the nozzle has been presented in section III.A when discussing the turbulence triggering model. It has been verified that the flow obtained for the full simulation collapses with the one presented previously, which ensures the convergence of the above-discussed flow fields and the validity of the corresponding discussions. In this section, focus is therefore given in the flow downstream of the nozzle exhaust.

\section{V.A. Recirculation bubble at nozzle exit}

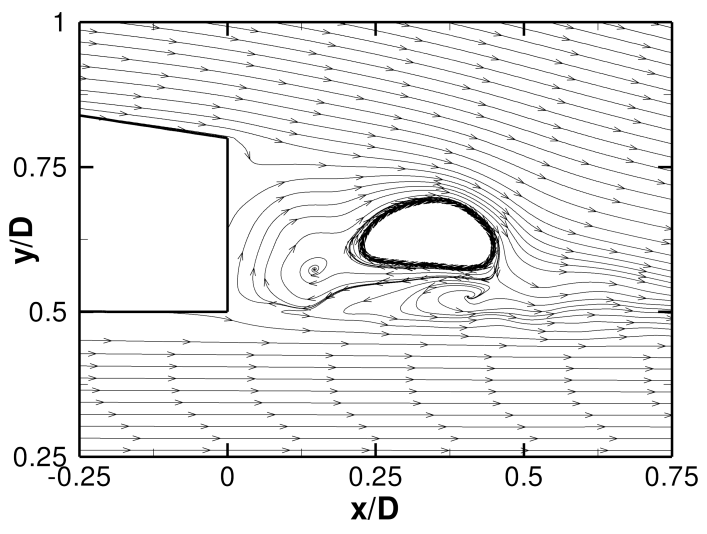

Figure 12. Streamlines downstream of the nozzle lip. Baseline simulation.

A particularity of the nozzle used for the present simulations is its large lip thickness, requested to experimentally mount the PSJs on the nozzle. As illustrated in Fig. 12, this thickened lip generates a recirculation bubble downstream of the exhaust, at about $x / D \sim 0.3$, and whose diameter is roughly half the lip thickness.

\section{V.B. Flow fields on jet axis}

The simulated and experimental evolutions of the mean axial velocity on jet axis are represented in Fig. 13. The baseline simulation profile is in good agreement with the experimental data up to $5 D$ and is underestimated more downstream, essentially because the velocity starts decreasing sooner numerically, compared to the experiment, and also because of the higher rate of velocity decay in the simulation. As a consequence, the potential core length $x_{c}$, defined as the distance from the nozzle exit where the axial velocity is $90 \%$ of the jet exhaust velocity, $u_{x}\left(x=x_{c}\right)=0.9 U_{j}$, is underestimated numerically and decreases from $8.1 D$ experimentally to $6.3 D$ in the simulation. Such an underprediction of the potential core length has been observed in previous numerical studies ${ }^{26,38,46-48}$ and might be related to the turbulence level at nozzle exit. This turbulence level indeed drives the initial shear layer state (laminar, transitional or turbulent) and is has been shown experimentally that jets with initially transitional shear layers develop with a shorter potential core length than initially turbulent jets. ${ }^{49}$ Such a dependence of the core length with the initial turbulence level has been also observed numerically by Bogey \& Bailly. ${ }^{39}$ In the present simulation, despite the turbulence triggering procedure used, the initial turbulence level very likely underestimates the experimental turbulence level expected to be of several percents, ${ }^{35,36}$ which may explain the underestimation of the core length.

In the presence of PSJs, the simulated axial velocity starts decreasing at a slightly lower axial position, compared to the baseline configuration, which results in a reduction of the potential core length of $3 \%$, from $6.3 D$ to $6.1 D$. Such a core length reduction has been observed experimentally by Kim et al. ${ }^{14}$ using LAFPAs with a similar forcing Strouhal number. It is clear that the technology of the actuators is different between the PSJs and the LAFPAs and their action on the jet is expected to differ, but they both experimentally lead to a broadband noise increase for a low frequency forcing and similar flow modifications can be expected between the two control technologies. 


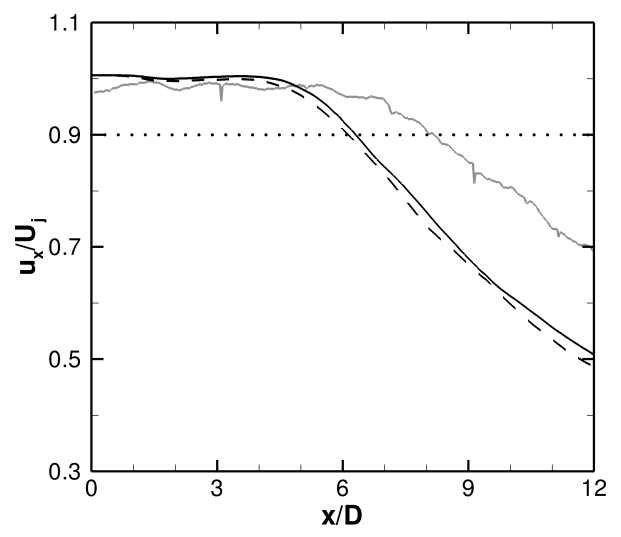

Figure 13. Evolution of mean axial velocity on jet axis. The dotted line represents the potential core length limit. Experiments without PSJs. Simulations - without and - - - with PSJs.

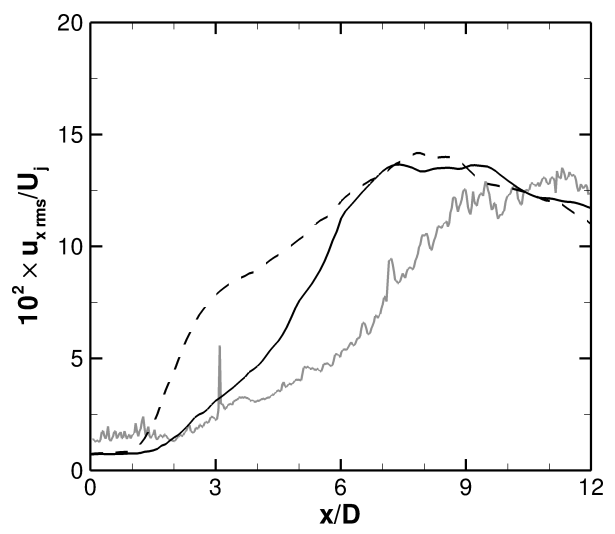

Figure 14. Evolution of turbulent axial velocity on jet axis. - Experiments without PSJs. Simulations — without and --- with PSJs.

Figure 14 displays the turbulent axial velocity profiles on jet axis. The experimental maximum turbulence level of $13 \%$ is very well reproduced numerically. This maximum level is however shifted towards the nozzle exit in the simulation, as a consequence of the numerical potential core length underestimation. In the presence of the control, one observes that the turbulence is increased in the first diameters of the jet. The maximum level is nevertheless very similar to that obtained for the baseline simulation.

\section{V.C. Flow evolution in the shear layer}

Numerically, the initially laminar shear layer state has been evidenced in section III.B. Experimentally, as visible in Fig. 15, the peak turbulence starts from a level of several percents at nozzle exit and rises up to $x / D \sim 2$ where it reaches a maximum value of about $15 \%$ of jet jet exhaust velocity $U_{j}$. Such a profile is characteristic of an initially turbulent shear layer. ${ }^{45}$ The different initial state of the shear layer between the experiment and the simulation is coherent with the numerical underestimation of the potential core length, discussed previously.

With PSJs, the reduction of the potential core length is coherent with the increase of the peak turbulent axial velocity in the vicinity of the nozzle exit, visible in Fig. 15. The peak turbulent velocity being representative of the turbulence levels in the shear layer, it indicates that the PSJs favour the growth of energetic structures in the mixing layer. For a given axial position, the peak turbulent velocity varies with the azimuth, as illustrated in Fig. 16. In this figure, the peak turbulent velocity is plotted for two azimuths corresponding to a position downstream of PSJs and a position between PSJs. The turbulence is noticeably higher downstream of the PSJs until $x / D=1$, after what the levels are identical between the two azimuths. The profile downstream of the PSJs especially exhibits a large overshoot for $x / D=0.1$. This position corresponds to the modelling of the actuators in the simulations and therefore represents the interaction of the main jet with the PSJs. 


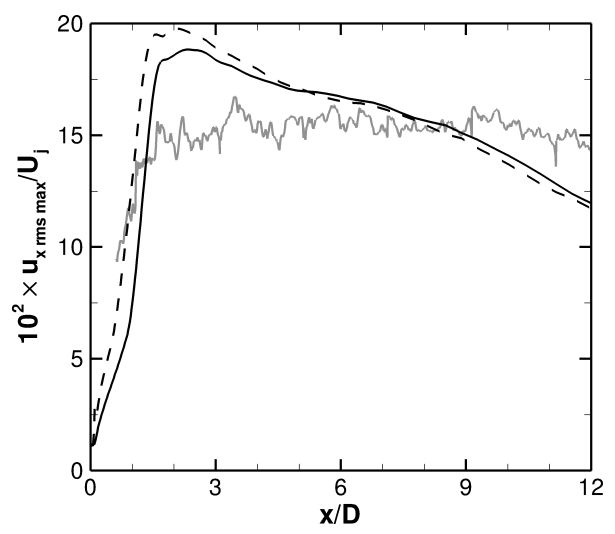

Figure 15. Axial evolution of peak turbulent axial velocity. — Experiments without PSJs. Simulations — without and -- with PSJs.

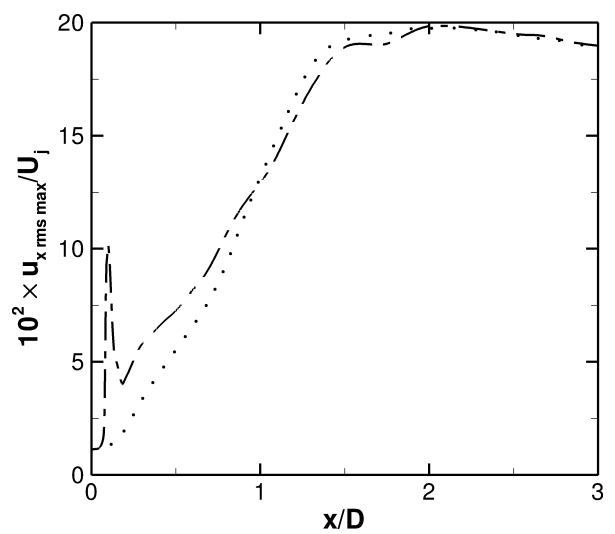

Figure 16. Detail of peak turbulent axial velocity evolution at nozzle exit. Simulation with PSJs at azimuthal location - - downstream of PSJs and . b between PSJs.

To figure out the origin of this turbulence increase in the presence of the PSJs, one needs to look at the vorticity snapshots downstream of the nozzle, represented in Fig. 17 for both simulations. In this figure, the time delay between two consecutive snapshots is $9 \times 10^{-5} \mathrm{~s}\left(0.074 / f_{F}\right)$ and, for the simulation with PSJs, the plane represented passes through the actuators. In Fig. 17 (b.1), one can see the fluid being injected by the PSJs. This is especially visible on the upper part of the shear layer, where it is indicated by an arrow. The fluid injection generates a large coherent vortex, first visible in Fig. 17 (b.2), that grows while being convected downstream. This structure, indicated by an arrow in the figures, remains very coherent and finally mixes with the jet own turbulence, as visible in Fig. 17 (b.5). The generation of such a vortex during the interaction of the PSJs with the main flow is in very good agreement with the experimental observations. It is reasonable to consider that this flow structure plays a key role in the flow and subsequent noise radiation modifications caused by the PSJs.

To complete the discussion of the flow evolution downstream of the nozzle lip, the evolution of the shear layer momentum thickness is represented is Fig. 18. The simulated momentum profile can be separated into three distinct stages. In the first stage, a very slow growth is observed until $x / D \sim 1$. It corresponds to a flow adjustment of the radial velocity profile from a boundary-layer profile, at nozzle exit, to a shear-layer profile inside the jet. The momentum thickness growth largely increases in the second stage, for $x / D \sim 2.0$. This region corresponds to the later stages of transition where large vortical structures are observed, see Fig 7. In the third stage, for $x / D>3.0$, a relatively linear growth with a lower growth rate of the momentum thickness is finally observed. This evolution in three stages is characteristic of initially laminar jets. It has been evidenced experimentally by Hussain \& Zedan ${ }^{50,51}$ and Husain \& Hussain ${ }^{52}$ and observed in the previous numerical works of Huet ${ }^{26}$ and Bogey \& Bailly, ${ }^{53}$ for instance. In the present simulation, however, the transition between the different stages occurs for a larger axial distance than in the above-mentioned experimental and numerical results. This difference might come from the larger initial momentum thickness $\delta_{\theta}(0)$ in the present simulation, that is a consequence of the triggering procedure used. Hussain \& Zedan ${ }^{50}$ 


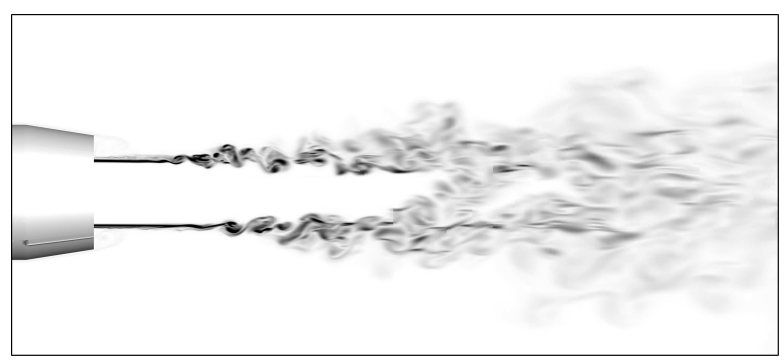

(a.1)

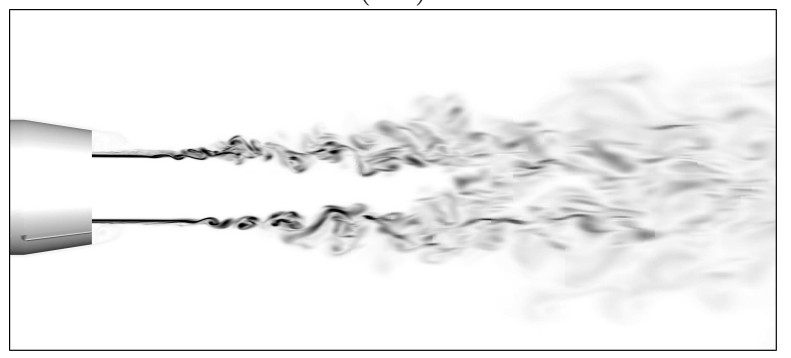

(a.2)

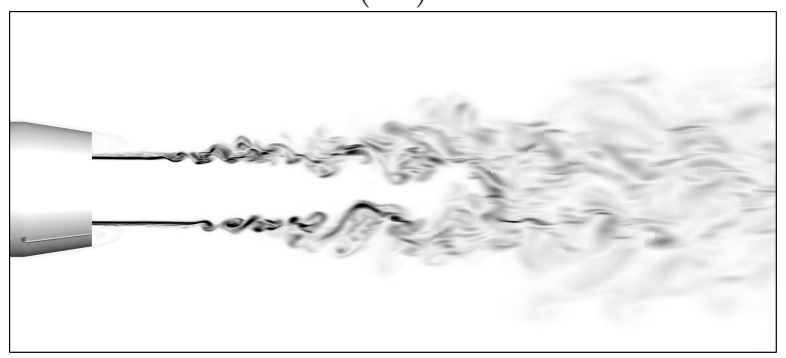

(a.3)

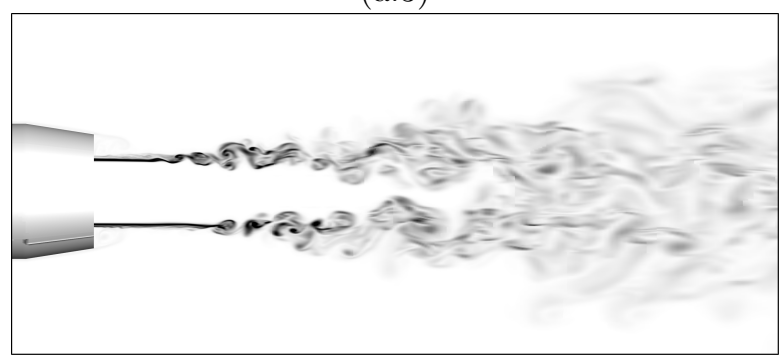

(a.4)

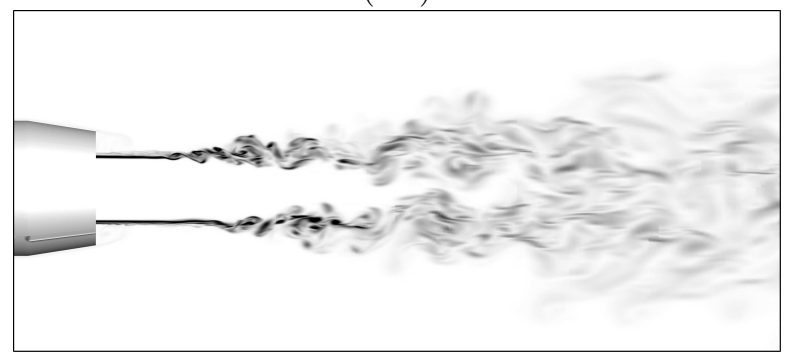

(a.5)

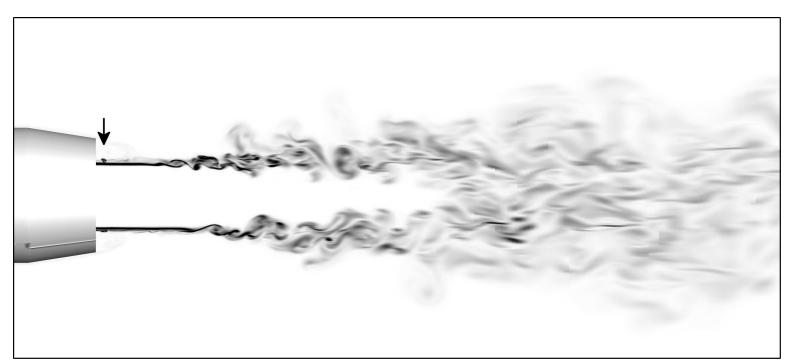

(b.1)

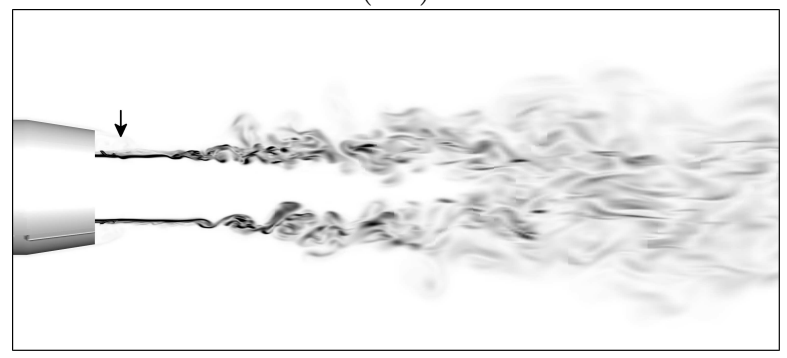

(b.2)

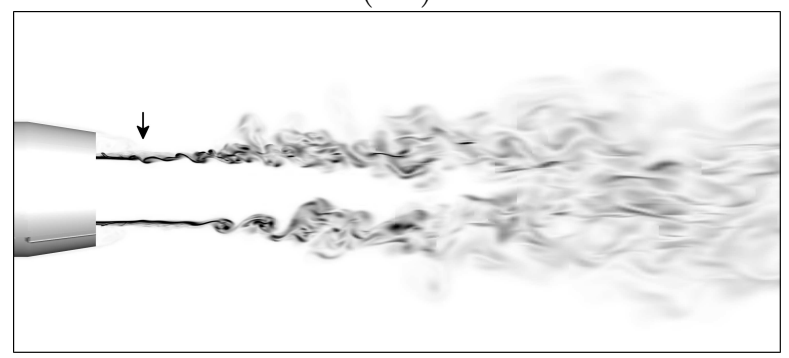

(b.3)

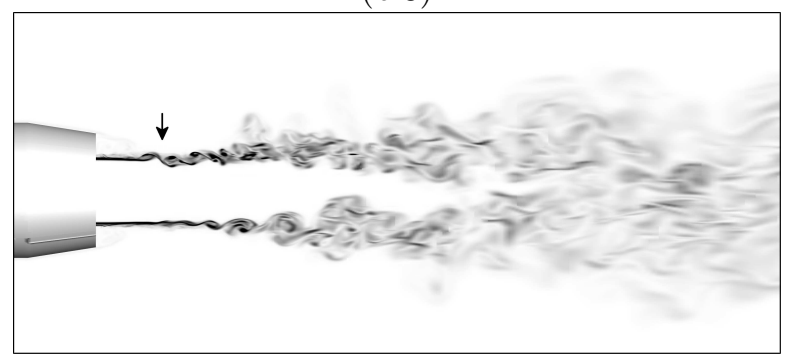

(b.4)

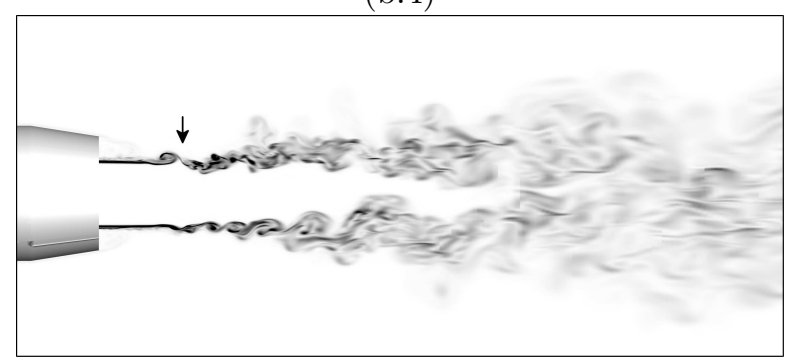

(b.5)

Figure 17. Vorticity snapshots (a) without and (b) with PSJs. The time delay between consecutive snapshots is $9 \times 10^{-5}$ s. 


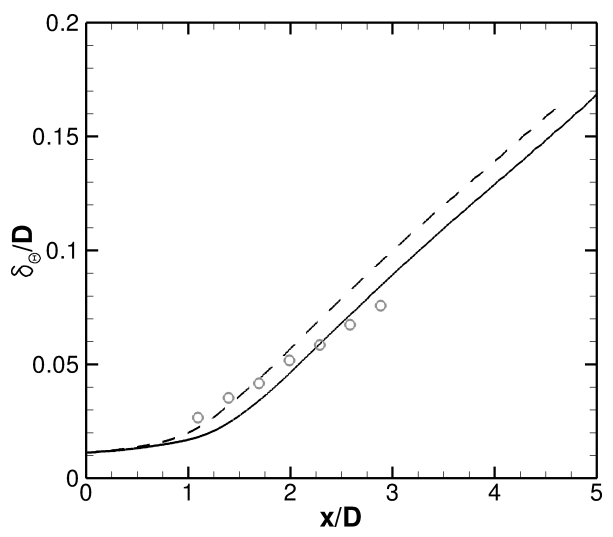

Figure 18. Axial evolution of the shear-layer momentum thickness. $\circ$ Experiments without PSJs. Simulations without and --- with PSJs.

indeed evidenced that the transition is shifted downstream of the nozzle exhaust when increasing the initial momentum thickness.

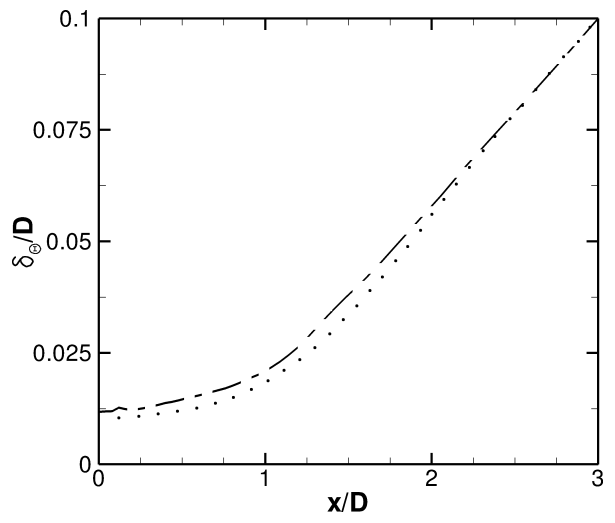

Figure 19. Detail of shear layer momentum thickness at nozzle exit. Simulation with PSJs at azimuthal location - downstream of PSJs and $\cdots$ between PSJs.

The structures generated by the PSJs and evidenced above may also be responsible of the larger shearlayer momentum thickness in the presence of the control, observed in Fig. 18. The control does not modify the growth rate ratio, but reduces the distance corresponding to the flow adjustment and leads to an earlier growth of the momentum thickness. The flow adjustment region being followed by the later stages of transition where large turbulent structures are observed, one can assume that PSJs favour the development of the turbulent structures of the jet. As for the peak turbulence evolution, the momentum thickness varies with the azimuthal position, see Fig. 19. The thickening is more important downstream of the PSJs compared to the azimuthal position between the PSJs, most likely because the large structures generated by the PSJs are azimuthally limited essentially to the region downstream of the actuators.

One may notice that the difference between the second and third stage of the momentum profile are not easy to distinguish. The lower growth rate in the third stage in evidenced in Fig. 20 where the spreading rate of the simulated momentum thickness $d \delta_{\theta} / d x$ is represented.

Numerically, the momentum thickness underestimates the experimental results for $x / D<2$, the opposite being observed for larger abscissas. Close to the nozzle exit, the underestimation may be explained by the flow adjustment observed in the initially laminar simulation, leading to a very low growth rate at least up to $x / D=1$. More downstream, the larger simulated growth rate explains the overestimation of the computed momentum thickness and, subsequently, the shorter potential core length.

\section{V.D. Cartographies of simulated turbulent axial velocity}

To give a more detailed insight into the simulated turbulence modifications caused by the PSJs, the cartographies of the turbulent axial velocity are reproduced in Fig. 21 for both simulations. For the configuration 


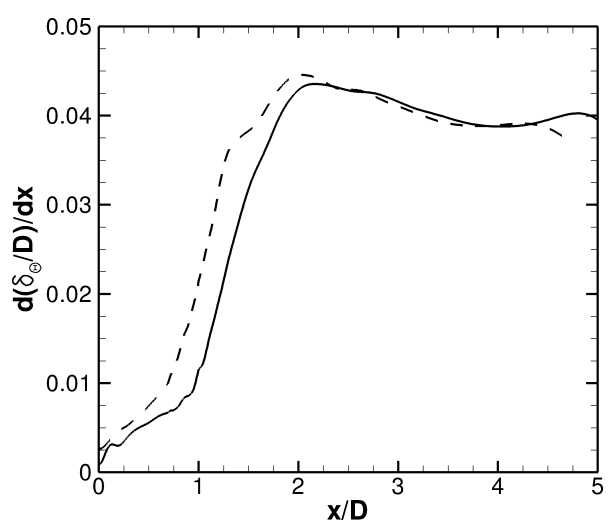

Figure 20. Spreading rate of the simulated shear-layer momentum thickness - without and - - with PSJs.

with PSJs, Fig. 21 (b) corresponds to a plane passing through the actuators whereas the plane passes between actuators in Fig. 21 (c).

In the presence of the PSJs, the differences between the two cartographies through and between PSJs are essentially located in the shear layer, in the first diameter downstream of the nozzle exit. More downstream, both planes exhibit similar levels which may indicate that the flow has recovered its axisymmetry for such axial positions.

Without control, the maximum turbulence levels $u_{x} r m s / U_{j}>0.18$ are observed along the shear layer, for an axial position between $2 D$ and $3.5 D$. This zone extends to $4 D$ in the presence of the PSJs and is radially more important, as a consequence of a global increase of the turbulence in the presence of the control. More downstream, very limited differences are observed between the two simulations and the alterations can be related to the different potential core length in the two simulations solely. The turbulence modifications caused by the control are therefore limited and essentially located in the vicinity of the actuators.

\section{Radiated noise}

In this section, the radiated noise simulated for both configurations is discussed and compared to the experiments. The time signals are stored on the surfaces for $70.20 \mathrm{~ms}$. Due to transients at the beginning and at the end of the radiation, the physical pressure time histories are available for $66.114 \mathrm{~ms}$. As previously mentionned in section II.C, it has been verified that the computed pressure signals do not depend on the position of the storage surface for frequencies below $7 \mathrm{kHz}$. Simulated pressure data presented in this section therefore correspond to one reference surface only.

\section{VI.A. Baseline configuration}

In a first step, the baseline simulated noise is compared to the measurement performed in the anechoic facility of the Centre Acoustique, LMFA - École Centrale de Lyon, to assess the capacity of the simulation to reproduce the experimental data in the absence of control. Experimental and simulated power spectral densities at observation angles of $30^{\circ}$ and $90^{\circ}$ are reproduced in Fig. 22.

At $30^{\circ}$, the simulation reproduces quite well the experimental spectrum. With the exception of the noise increase observed numerically for the low frequencies and that was shown to be spurious noise caused by the presence of turbulent spots on the storage surface, simulated levels are quantitatively similar to those obtained experimentally below $1000 \mathrm{~Hz}$. Between $1000 \mathrm{~Hz}$ and $7000 \mathrm{~Hz}$, computed levels overestimate the experimental data with a difference lying between $1 \mathrm{~dB}$ and $3 \mathrm{~dB}$, depending of the frequency considered. These excessive high noise levels observed numerically are essentially the consequence of the slight overprediction of the frequency of maximum level in the computation. Experimentally, this frequency is $f=1000 \mathrm{~Hz}\left(S t_{D}=\right.$ $\left.f_{D} / U_{j}=0.17\right)$ whereas a value of $1300 \mathrm{~Hz}\left(S t_{D}=f_{D} / U_{j}=0.22\right)$ is obtained in the simulation. A similar shift of the maximum level towards the high frequencies has been observed numerically by Bogey \& Bailly. ${ }^{53}$ It is all the more pronounced that the inlet turbulence level is low and seems therefore to be related to the initially laminar shear layer state. The consequences of the laminar mixing layer on noise are more visible at $90^{\circ}$ and will be discussed in more details in the next paragraph. Above $7000 \mathrm{~Hz}$, simulated levels collapse and 


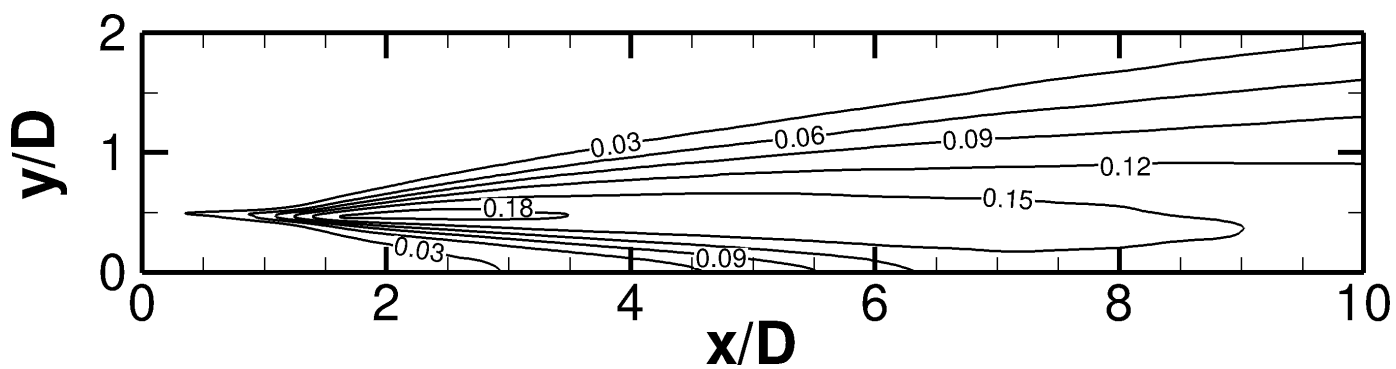

(a) without PSJs

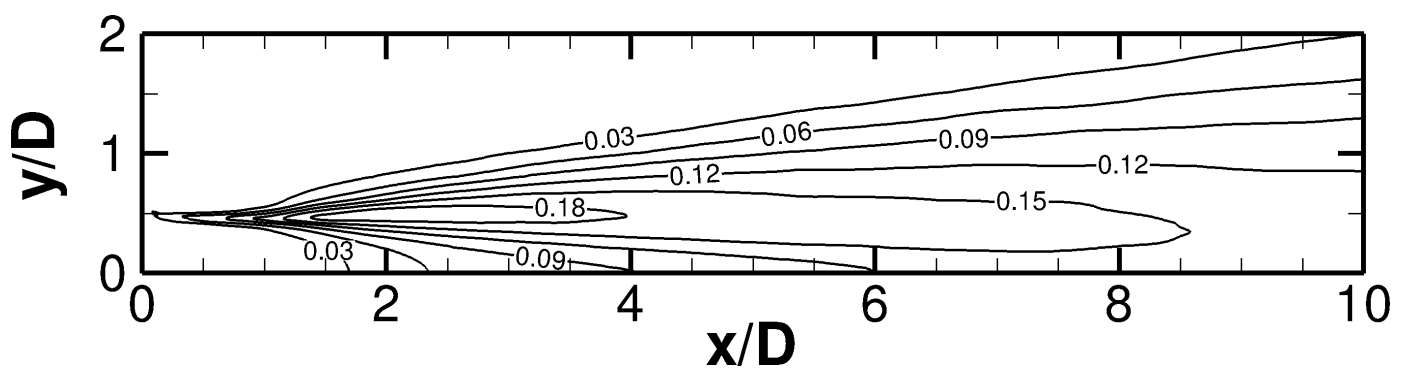

(b) through PSJs

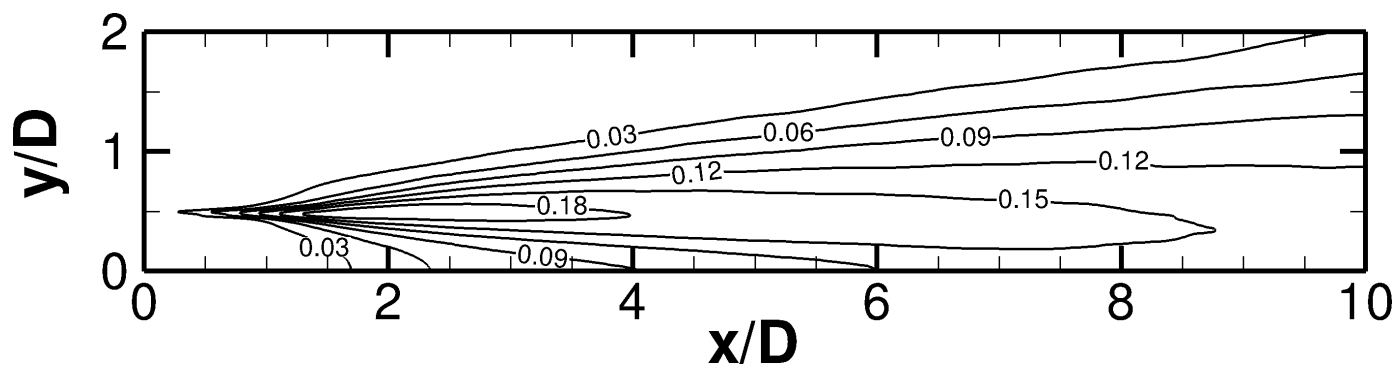

(c) between PSJs

Figure 21. Cartographies of simulated turbulent axial velocity $u_{x r m s} / U_{j}$.

strongly underestimate the experiments. This latter frequency corresponding to the grid cut-off frequency, these very low numerical levels are therefore a consequence of the numerical dissipation in the aerodynamic simulations.

The higher noise levels observed numerically at $30^{\circ}$ are also visible at the observation angle of $90^{\circ}$. One can especially notice the spurious noise caused by the turbulent structures present on the storage surface for the very low frequencies, more pronounced that at $30^{\circ}$. Medium frequency noise also presents an overestimation compared to the experiments that reaches $4 \mathrm{~dB}$ for $f \sim 3000 \mathrm{~Hz}$. This overestimation is to a large extent the consequence of the significant overestimation of the peak frequency, predicted for $f=2300$ $\mathrm{Hz}\left(S t_{D}=0.38\right)$ numerically in comparison to $f=1300 \mathrm{~Hz}\left(S t_{D}=0.22\right)$ in the experiments.

The origin of this overestimation of the peak frequency has been evidenced experimentally by Zaman ${ }^{35,36}$ and discussed in the numerical studies of Bogey et al. ${ }^{38}$ and Huet ${ }^{26}$ amongst others. It is a consequence of the initially laminar state of the shear layer that leads to the generation of an additional noise source in the medium frequency range, compared to initially turbulent jets, that essentially radiates near $90^{\circ}$. In initially laminar jets, large coherent vortices take birth in the early stage of the shear layer and grow before pairing with one another and radiating noise essentially in the direction normal to the jet. Zaman observed that the peak frequency in the far field is obtained for $S t_{\theta} \sim 0.006$, where $S t_{\theta}=f \delta_{\theta}(0) / U_{j}$ is the Strouhal number based on the initial shear-layer momentum thickness $\delta_{\theta}(0)$. Noticing that this frequency is half the value of the roll-up frequency ${ }^{36,54}$ he concluded that the first stage of pairing is the source of the additional noise.

In the present simulation without PSJs, the initial momentum thickness $\delta_{\theta}(0)$ is $0.0115 D$. The simulated peak frequency being $2300 \mathrm{~Hz}$ at $90^{\circ}$, it leads to $S t_{\theta} \sim 0.0045$. This Strouhal number is close to the value observed experimentally for initially laminar jets and may confirm the vortex pairings as the origin of this additional noise source in the medium frequency range for the present simulation. 


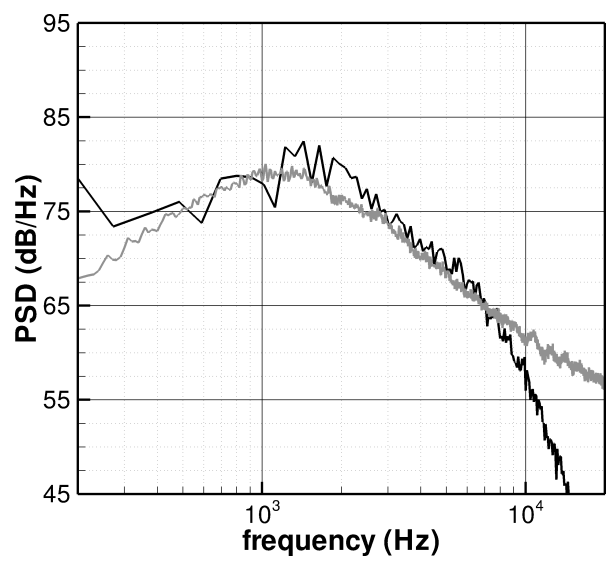

(a) $30^{\circ}$

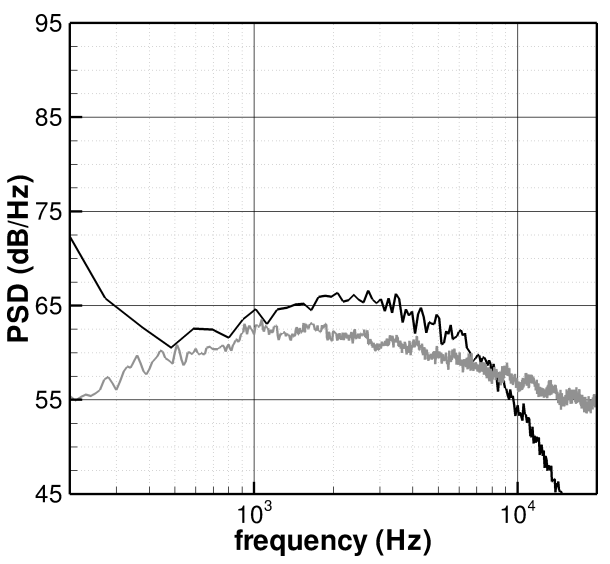

(b) $90^{\circ}$

Figure 22. Power spectral densities at observer locations $40 D$ (2 meters) from the nozzle exit. Baseline configuration. experiments; - simulation.

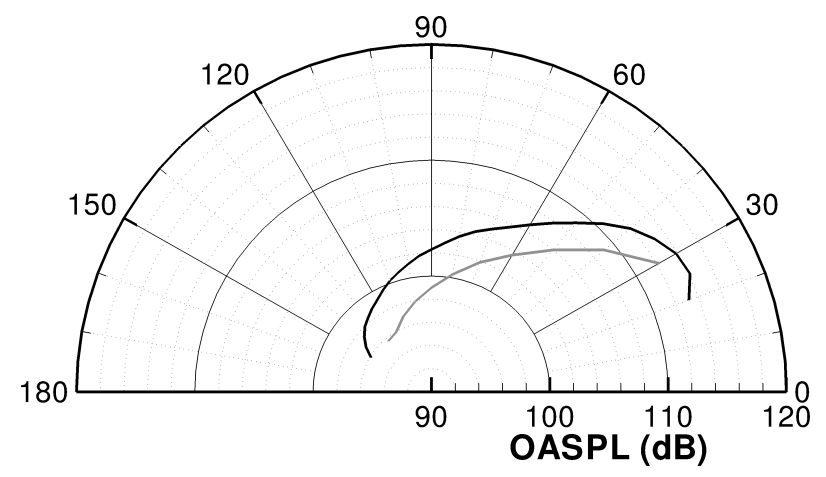

Figure 23. Overall sound pressure levels integrated obtained $40 D$ (2 meters) from the nozzle exit. Baseline configuration. Integration is performed for the frequency bandwidth $[200 \mathrm{~Hz} ; 7000 \mathrm{~Hz}]$. — experiments; - simulation.

The sound pressure levels obtained at $40 D$ are reproduced in Fig. 23. The experimental directivity pattern is well reproduced numerically, with especially a very good prediction of the maximum radiation angle in the fore direction. A slight overestimation of the sound pressure levels is nevertheless observed in the simulation for all observation angles, varying from $1.7 \mathrm{~dB}$ at $30^{\circ}$ to $3.3 \mathrm{~dB}$ at $90^{\circ}$. It is a consequence of the spurious low-frequency noise and of the overestimation of the medium-frequency levels in the computations, previously observed in the power spectral densities in Fig. 22. This discrepancy remains however relatively small and illustrates the correct representation of the jet flow development and noise generation and radiation in the present simulation.

\section{VI.B. PSJs action on radiated noise}

In the previous paragraph, the capacity of the simulations to accurately reproduce the noise radiated by the jet has been assessed for the baseline configuration. The modification of the far-field noise by the control of the jet with PSJs is now investigated numerically and compared to experimental results.

Experimental power spectral densities at $30^{\circ}$ and $90^{\circ}$ without and with PSJs are reproduced in Fig. 24. The results represented at these two angles are representative of the action of the PSJs on radiated noise at all observer locations. In the presence of the control, one first observes the presence of tonal noises for some harmonics of the forcing frequency $f_{F}=820 \mathrm{~Hz}$. The control also increases the broadband noise of 1 $\mathrm{dB} / \mathrm{Hz}$ in the low frequency range and between $1.5 \mathrm{~dB} / \mathrm{Hz}$ and $2 \mathrm{~dB} / \mathrm{Hz}$ for larger frequencies. These results are very similar to those obtained by Samimy et al. ${ }^{13}$ and Kastner et al..$^{15}$ for the control of a subsonic isothermal jet with LAFPAs. Despite some technological differences between the PSJs and the LAFPAs, the two control systems therefore seem to have a very similar action on the jet flow and related noise sources development.

The simulated power spectral densities without and with control are illustrated in Fig. 25. The low 


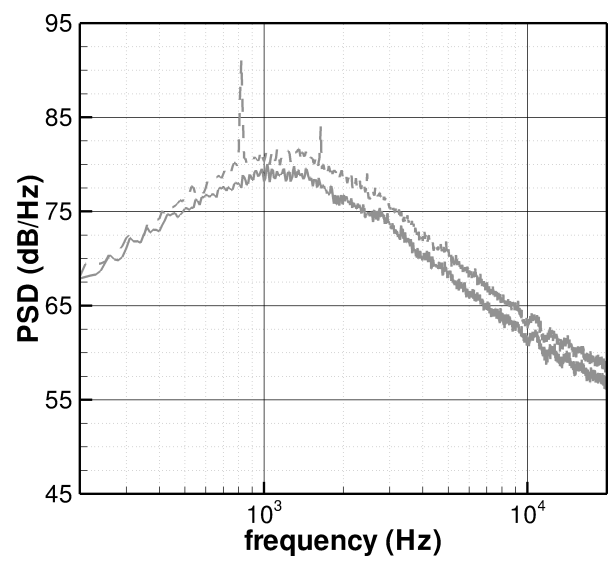

(a) $30^{\circ}$

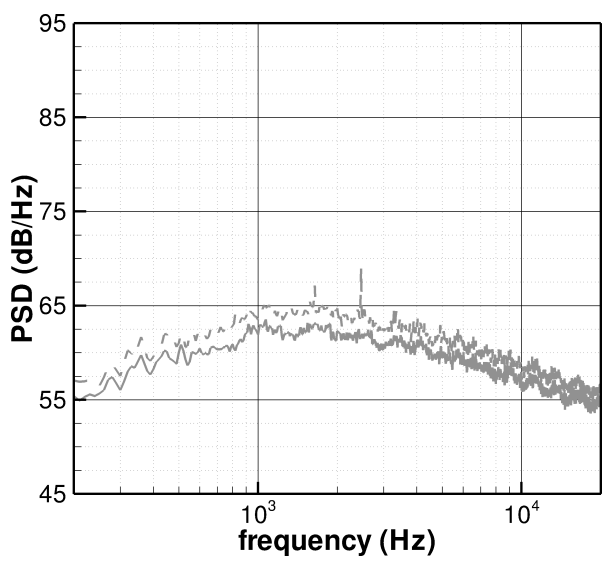

(b) $90^{\circ}$

Figure 24. Power spectral densities at observer locations $40 D$ (2 meters) from the nozzle exit. Experimental data without and - - - with PSJs.

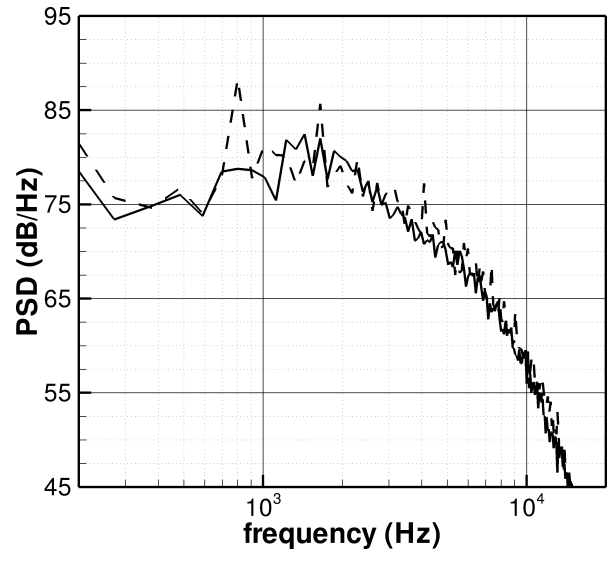

(a) $30^{\circ}$

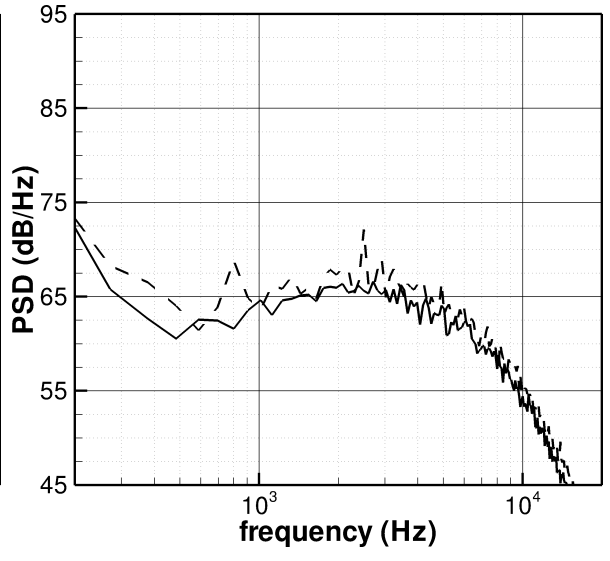

(b) $90^{\circ}$

Figure 25. Power spectral densities at observer locations $40 D$ (2 meters) from the nozzle exit. Simulated data without and - - - with PSJs.

frequency part of the spectra being contaminated by the presence of turbulent structures on the radiation surfaces, the noise variation caused by the PSJs will not be discussed for such long period phenomena. As in the experiments, tonal noises are visible for frequencies multiple of the forcing frequency. With the exception of the medium frequency range for the observer position of $30^{\circ}$, where no significant variations of the noise are visible with the presence of the control, the simulated action of the PSJs on the broadband noise fairly well reproduces the experiments. At $30^{\circ}$ and for frequencies above $3 \mathrm{kHz}$, PSJs increase the noise of about $1.5 \mathrm{~dB} / \mathrm{Hz}$. A similar increase of $1.5 \mathrm{~dB} / \mathrm{Hz}$ is observed at $90^{\circ}$ for both medium and high frequency noise, in very good agreement with the experimental data.

The sound pressure levels obtained at $40 D$ without and with PSJs are reproduced in Fig. 26 for the experiments and in Fig. 27 for the simulations. On both figures, the sound pressure levels obtained after a filtering of the tonal noises for the configuration with PSJs is also represented. These levels are reproduced to illustrate the action of the PSJs on the broadband noise of the jet, without contamination of the levels with the energetic tonal noises, as in previous literature results. ${ }^{13,55,56}$ Experimentally, PSJs increase the noise of about $2 \mathrm{~dB}$ for all observation angles. It is worth mentioning that this value is very similar to the experimental results obtained by Samimy et al. ${ }^{13}$ using LAFPAs with the same forcing frequency, despite technological differences and a different setup of the actuators. The principal cause of this noise increase is the raise of the broadband noise in the presence of the control. Tonal noises only have a negligible contribution on the global pressure levels; indeed, the evaluation of the OASPL after filtering the tonal noises showed a level difference below $0.1 \mathrm{~dB}$ for most of the observation angles.

Numerically, the contribution of the tonal noises to the integrated pressure levels in the presence of the 


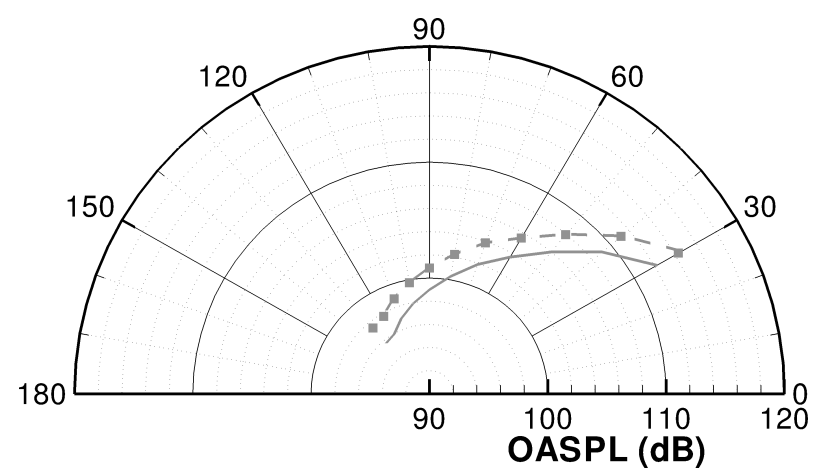

Figure 26. Overall sound pressure levels integrated obtained $40 D$ (2 meters) from the nozzle exit. Experimental data without PSJs, - - with PSJs, with PSJs and filtered tones. Integration is performed for the frequency bandwidth $[200 \mathrm{~Hz} ; 7000 \mathrm{~Hz}]$.

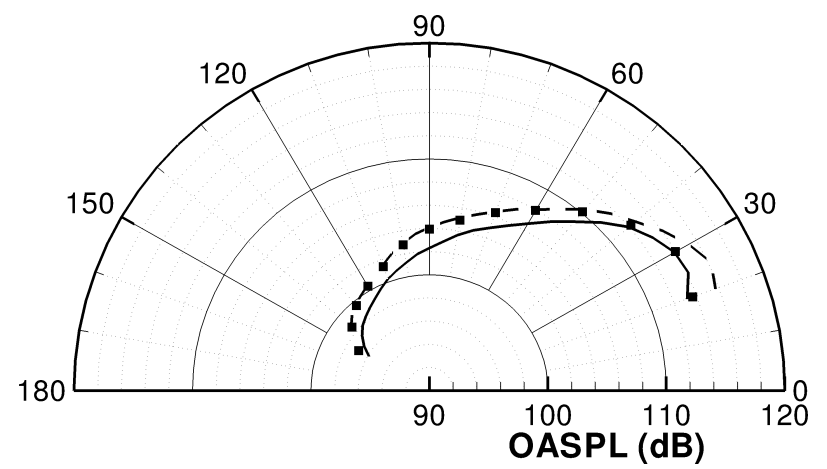

Figure 27. Overall sound pressure levels integrated obtained $40 \mathrm{D}$ (2 meters) from the nozzle exit. Simulated data without PSJs, - - with PSJs, $\square$ with PSJs and filtered tones. Integration is performed for the frequency bandwidth [200 Hz; $7000 \mathrm{~Hz}]$.

PSJs is more visible at fore angles when compared to the experiments. This contribution of the tones to the OASPL stays however limited to observation angles below $50^{\circ}$. The broadband noise variation caused by the PSJs lead to a noise increase that varies between $0.1 \mathrm{~dB}$ at $30^{\circ}$ and about $1.5 \mathrm{~dB}$ above $50^{\circ}$, this value being in very good agreement with the experiments. The lower noise increase observed at fore angles is caused by the absence of noise modification in the medium frequency range for this observer location, as previously observed in Fig. 25.

\section{Conclusion}

The large-eddy simulations of a single stream, isothermal jet with a Reynolds number of $10^{6}$ and a Mach number of 0.9 are presented. The control of the jet is especially investigated numerically with the use of 12 plasma synthetic jets (PSJs), located at nozzle lip and acting with a forcing frequency of $820 \mathrm{~Hz}\left(S t_{F}=0.14\right)$. For the sake of simplicity, the PSJs are not discretized in the numerical grid but are modelled with source terms in the equations of mass, momentum and energy for specific cells corresponding to the locations of the actuators. This modelling is aimed to match PSJs reference velocity and temperature. The objective of such a modelling is to reproduce the main characteristic of the actuators, namely the momentum injection, which is thought to be of prime importance in the jet flow control.

The agreement between the reference simulation, without control, and the experimental data is found to be satisfactory for both flow and noise. Despite the low turbulence level at nozzle exit in the simulation, the flow development reproduces quite well the experimental results. Numerically, the PSJs are found to generate large, coherent vortices in the jet shear layer and to favour the generation of turbulence. From the acoustics point of view, all presented results illustrate the capacity of the numerical simulations to reproduce the noise generated by experimental turbulent jets, without and with control. The baseline configuration is especially well reproduced with a limited overestimation of the integrated levels, below $2 \mathrm{~dB}$ at fore angles and of about $3 \mathrm{~dB}$ at aft angles. In the presence of the PSJs, the experimental noise modifications are 
accurately reproduced numerically, especially above $50^{\circ}$ with a similar raise of the broadband noise levels and an increase of the integrated levels for both experiments and simulations.

These results demonstrate the reliability of the present methodology to investigate the flow and noise control of jets with PSJs. In the studies to come, the current approach will be used as a numerical tool to supplement the results obtained in the test facility, to provide a better understanding of the action of the PSJs on the flow and to help go through the technological limitations of the small scale actuators. Indeed, present investigations illustrate the capacity of the PSJs to increase the broadband noise of jets at low forcing frequencies. A noise reduction is thought to be observed for larger forcing frequencies, but such a control cannot be achieved experimentally in the present days. Future simulations will focus on simulating such configurations in order to evidence the interest of PS.Js in jet noise reduction for full scale engines.

\section{Acknowledgments}

This work was conducted within the project ORINOCO, a collaborative project between the European Union and Russia coordinated by F. Cléro and V. Kopiev. The author is very grateful to O. Léon and D. Caruana from Onera for providing the aerodynamic experimental data and to D. Juvé, T. Castelain and P. Souchotte from LMFA - École Centrale de Lyon for the acoustic measurements.

\section{References}

${ }^{1}$ Laufer, J., Schlinker, R., and Kaplan, R. E., "Experiments on supersonic jet noise," AIAA Journal, Vol. 14, 1976, pp. 489-497.

${ }^{2}$ Fisher, M. J., Harper-Bourne, M., and Glegg, S. A. L., "Jet engine noise source location: the polar correlation technique," Journal of Sound and Vibration, Vol. 51, 1977, pp. 23-54.

${ }^{3}$ Bridges, J. and Brown, C. A., "Parametric testing of chevrons on single flow hot jets," Proceedings of the $10^{\text {th }}$ AIAA/CEAS Aeroacoustics Conference, No. AIAA 2004-2824, 2004.

${ }^{4}$ Alkislar, M. B., Krothapalli, A., and Butler, G., "The effect of streamwise vortices on the aeroacoustics of a Mach 0.9 jet," Journal of Fluid Mechanics, Vol. 578, 2007, pp. 139-169.

${ }^{5}$ Nesbitt, E. and Young, R., "Forward flight effects on chevron noise reduction," Proceedings of the $14^{\text {th }}$ AIAA/CEAS Aeroacoustics Conference, No. AIAA 2008-3065, 2008.

${ }^{6}$ Zaman, K. B. M. Q., "Effects of delta tabs on mixing and axis switching in jets from axisymmetric nozzles," Proceedings of the 32 $2^{\text {nd }}$ Aerospace Sciences Meeting and Exhibit, No. AIAA 1994-186, 1994.

${ }^{7}$ Bridges, J. and Wernet, M. P., "Turbulence measurements of separate flow nozzles with mixing enhancement features," Proceedings of the $8^{\text {th }}$ AIAA/CEAS Aeroacoustics Conference, No. AIAA 2002-2484, 2002.

${ }^{8}$ Tam, C. K. W. and Zaman, K. B. M. Q., "Subsonic jet noise from nonaxisymmetric and tabbed nozzles," AIAA Journal, Vol. 38, 2000, pp. 592-599.

${ }^{9}$ Castelain, T., Sunyach, M., Juvé, D., and Béra, J.-C., "Jet noise reduction by impinging microjets: an aerodynamic investigation testing microjet parameters," Proceedings of the $13^{\text {th }}$ AIAA/CEAS Aeroacoustics Conference, No. AIAA 2007$3419,2007$.

${ }^{10}$ Arakeri, V. H., Krothapalli, A., Siddavaram, V., Alkislar, M. B., and Lourenco, L. M., "On the use of microjets to suppress turbulence in a Mach 0.9 axisymmetric jet," Journal of Fluid Mechanics, Vol. 490, 2003, pp. 75-98.

${ }^{11}$ Castelain, T., Sunyach, M., Juvé, D., and Béra, J.-C., "Jet-noise reduction by impinging microjets: an acoustic investigation testing microjet parameters," AIAA Journal, Vol. 46, 2008, pp. 1081-1087.

${ }^{12}$ Ragaller, P. A., Annaswamy, A. M., Greska, B., and Krothapalli, A., "Supersonic jet noise reduction via pulsed microjet injection," Proceedings of the 15th AIAA/CEAS Aeroacoustics Conference, No. AIAA 2009-3224, 2009.

${ }^{13}$ Samimy, M., Kim, J.-H., Kastner, J., Adamovich, I., and Utkin, Y., "Active control of a Mach 0.9 jet for noise mitigation using plasma actuators," AIAA Journal, Vol. 45, 2007, pp. 890-901.

${ }^{14} \mathrm{Kim}$, J.-H., Kastner, J., and Samimy, M., "Active control of a high Reynolds number mach 0.9 axisymmetric Jet," $A I A A$ Journal, Vol. 47, 2009, pp. 116-128.

${ }^{15}$ J. Kastner, J.-H. Kim, M. S., "A study of the correlation of large-scale structure dynamics and far-field radiated noise in an excited Mach 0.9 jet," International Journal of Aeroacoustics, Vol. 8, 2009, pp. 231-259.

${ }^{16}$ Kearney-Fischer, M., Kim, J.-H., and Samimy, M., "Control of a high Reynolds number Mach 0.9 heated jet using plasma actuators," Physics of Fluids, Vol. 21, 2009, pp. 095101.

${ }^{17}$ Kearney-Fischer, M., Kim, J.-H., and Samimy, M., "Noise control of a high Reynolds number Mach 0.9 heated jet using plasma actuators," Proceedings of the 15 th AIAA/CEAS Aeroacoustics Conference, No. AIAA 2009-3188, 2009.

${ }^{18}$ Caruana, D., Barricau, P., Hardy, P., Cambronne, J.-P., and Belinger, A., "The "Plasma Synthetic Jet" actuator. Aerothermodynamic characterization and first flow control applications," Proceedings of the $4^{\text {th }}$ AIAA Aerospace Sciences Meeting, No. AIAA 2009-1307, 2009.

${ }^{19}$ Hardy, P., Barricau, P., Belinger, A., Caruana, D., Cambronne, J.-P., and Gleyzes, C., "Plasma Synthetic Jet for flow control," Proceedings of the $40^{\text {th }}$ Fluid Dynamics Conference and Exhibit, No. AIAA 2010-5103, 2010.

${ }^{20}$ Belinger, A., Hardy, P., Barricau, P., Cambronne, J.-P., and Caruana, D., "Influence of the energy dissipation rate in the discharge of a plasma synthetic jet actuator," Journal of Physics D: Applied Physics, Vol. 44, 2011, pp. 365201. 
${ }^{21}$ Caruana, D., Rogier, F., Dufour, G., and Gleyzes, C., "The plasma synthetic jet actuator, physics, modeling and flow control application on separation," Aerospace Lab, Vol. 6, 2013, pp. AL06-10.

${ }^{22}$ Dufour, G., Hardy, P., Quint, G., and Rogier, F., "Physics and models for plasma synthetic jets," International Journal of Aerodynamics, Vol. 3, 2013, pp. 47-70.

${ }^{23}$ Jordan, P., Gervais, Y., Valière, J.-C., and Foulon, H., "Final results from single point measurements, Project deliverable D3.4, JEAN-EU ${ }^{\text {th }}$ Framework Programme, G4RD-CT2000-0313," Tech. rep., Laboratoire d'Études Aérodynamiques, 2002.

${ }^{24}$ Jordan, P., Gervais, Y., Valière, J.-C., and Foulon, H., "Results from Acoustic Field Measurements, Project deliverable D3.6, JEAN-EU ${ }^{\text {th }}$ Framework Programme, G4RD-CT2000-0313," Tech. rep., Laboratoire d'Études Aérodynamiques, 2002.

${ }^{25}$ Huet, M., Rahier, G., and Vuillot, F., "Simulation of flow control with microjets for subsonic jet noise reduction," Applied aerodynamics, edited by J. C. Lerner, chap. 5, Intech, 2012, pp. 79-106.

${ }^{26}$ Huet, M., "Influence of boundary layers resolution on heated,subsonic, high Reynolds number jet flow and noise," Proceedings of the $19^{\text {th }}$ AIAA/CEAS Aeroacoustics Conference, No. AIAA 2013-2141, 2013.

${ }^{27}$ Dorey, L.-H., Tessé, L., Bertier, N., and Dupoirieux, F., "A strategy for modeling soot formation and radiative transfer in turbulent flames," Proceedings of the $14^{\text {th }}$ International Heat Transfer Conference, No. IHTC 14-22819, 2010.

${ }^{28}$ Dupoirieux, F. and Bertier, N., "The models of turbulent combustion in the CHARME Solver of CEDRE," Aerospace Lab, Vol. 2, 2011, pp. AL02-02.

${ }^{29}$ Guillou, F. and Chedevergne, F., "Internal turbine blade cooling simulation: advanced models assessment on ribbed configurations," Proceedings of the $8^{\text {th }}$ Thermal Engineering Joint Conference, No. AJTEC 2011-44295, 2011.

${ }^{30}$ Bodard, G., Bailly, C., and Vuillot, F., "Matched hybrid approaches to predict jet noise by using large-eddy simulation," Proceedings of the $15^{\text {th }}$ AIAA/CEAS Aeroacoustics Conference, No. AIAA 2009-3316, 2009.

${ }^{31}$ Courbet, B., Benoit, C., Couaillier, V., Haider, F., Le Pape, M.-C., and Péron, S., "Space discretization methods," Aerospace Lab, Vol. 2, 2012, pp. AL02-06.

${ }^{32}$ Smagorinsky, J., "General circulation experiments with the primitive equations," Monthly Weather Review, Vol. 91, 1963, pp. $99-164$

${ }^{33}$ Vuillot, F., Lupoglazoff, N., and Huet, M., "Effect of a pylon on double stream jet noise from hybrid CAA computations," Proceedings of the $16^{\text {th }}$ AIAA/CEAS Aeroacoustics Conference, No. AIAA 2010-4029, 2010.

${ }^{34}$ Vuillot, F., Lupoglazoff, N., and Huet, M., "Effect of chevrons on double stream jet noise from hybrid CAA computations," Proceedings of the $49^{\text {th }}$ AIAA Aerospace Sciences Meeting, No. AIAA 2011-1154, 2011.

${ }^{35}$ Zaman, K. B. M. Q., "Far-field noise of a subsonic jet under controlled excitation," Journal of Fluid Mechanics, Vol. 152, 1985, pp. 83-111.

${ }^{36}$ Zaman, K. B. M. Q., "Effect of initial condition on subsonic jet noise," AIAA Journal, Vol. 23, 1985, pp. 1370-1373.

${ }^{37}$ Zaman, K. B. M. Q., "Effect of initial boundary-layer state on subsonic jet noise," AIAA Journal, Vol. 50, 2012, pp. $1784-1795$.

${ }^{38}$ C. Bogey, S. Barré, C. B., "Direct computation of the noise generated by subsonic jets originating from a straight pipe nozzle," International Journal of Aeroacoustics, Vol. 7, 2008, pp. 1-22.

${ }^{39}$ C. Bogey, O. Marsden, C. B., "Influence of initial turbulence level on the flow and sound fields of a subsonic jet at a diameter-based Reynolds number of $10^{5}$," Journal of Fluid Mechanics, Vol. 701, 2012, pp. 352-385.

${ }^{40}$ D. J. Bodony, S. K. L., "On using large-eddy simulation for the prediction of noise from cold and heated turbulent jets," Physics Of Fluids, Vol. 17, 2005, pp. 085103.

${ }^{41}$ Bogey, C., Bailly, C., and Juvé, D., "Noise investigation of a high subsonic, moderate Reynolds number jet using a compressible Large Eddy Simulation," Theoretical and Computational Fluid Dynamics, Vol. 16, 2003, pp. 273-297.

${ }^{42}$ Ffowcs Williams, J. E. and Hawkings, D. L., "Sound generation by turbulence and surfaces in arbitrary motion," Philosophical Transactions for the Royal Society of London, Vol. A264, 1969, pp. 321-342.

${ }^{43}$ Rahier, G., Prieur, J., Vuillot, F., Lupoglazoff, N., and Biancherin, A., "Investigation of integral surface formulations for acoustic post-processing of unsteady aerodynamic jet simulations," Aerospace Science and Technology, Vol. 8, 2004, pp. 453-467.

${ }^{44}$ Huet, M., Fayard, B., Rahier, G., and Vuillot, F., "Numerical investigation of the micro-jets efficiency for jet noise reduction," Proceedings of the $15^{\text {th }}$ AIAA/CEAS Aeroacoustics Conference, No. AIAA 2009-3127, 2009.

${ }^{45}$ Castelain, T., Contrôle de jet par microjets impactants. Mesure de bruit rayonné et analyse aérodynamique, Ph.D. thesis, École Centrale de Lyon, 2006.

${ }^{46}$ Andersson, N., Eriksson, L.-E., and Davidson, L., "Large-eddy simulation of subsonic turbulent jets and their radiated sound," AIAA Journal, Vol. 43, 2005, pp. 1899-1912.

${ }^{47}$ DeBonis, J. R. and Scott, J. N., "Large-eddy simulation of a turbulent compressible round jet," AIAA Journal, Vol. 40, 2002, pp. 1346-1354.

${ }^{48}$ Lupoglazoff, N., Biancherin, A., Vuillot, F., and Rahier, G., "Comprehensive 3D unsteady simulations of subsonic and supersonic hot jet flow-fields. Part I: aerodynamic analysis," Proceedings of the $8^{\text {th }}$ AIAA/CEAS Aeroacoustics Conference, No. AIAA 2002-2599, 2002.

${ }^{49}$ Raman, G., Rice, E. J., and Reshotko, E., "Mode spectra of natural disturbances in a circular jet and the effect of acoustic forcing," Experiments In Fluids, Vol. 17, 1994, pp. 415-426.

${ }^{50}$ Hussain, A. K. M. F. and Zedan, M. F., "Effects of the initial condition on the axisymmetric free shear layer: Effects of the initial momentum thickness," Physics Of Fluids, Vol. 21, 1978, pp. 1100-1112.

${ }^{51}$ Hussain, A. K. M. F. and Zedan, M. F., "Effects of the initial condition on the axisymmetric free shear layer: Effect of the initial fluctuation level," Physics Of Fluids, Vol. 21, 1978, pp. 1475-1481.

${ }^{52}$ Husain, Z. D. and Hussain, A., "Axisymmetric mixing layer: influence of the initial and boundary conditions," AIAA Journal, Vol. 17, 1979, pp. 48-55.

${ }^{53}$ Bogey, C. and Bailly, C., "Influence of nozzle-exit boundary-layer conditions on the flow and acoustic fields of initially laminar jets," Journal of Fluid Mechanics, Vol. 663, 2010, pp. 507-538. 
${ }^{54}$ Zaman, K. B. M. Q. and Hussain, A. K. M. F., "Turbulence suppression in free shear flows by controlled excitation," Journal of Fluid Mechanics, Vol. 103, 1981, pp. 133-159.

${ }^{55}$ Moore, C. J., "The role of shear-layer instability waves in jet exhaust noise," Journal of Fluid Mechanics, Vol. 80, 1977, pp. 321-367.

${ }^{56}$ K. K. Ahuja, D. F. B., "Tone excited jets, part 4: acoustic measurements," Journal of Sound and Vibration, Vol. 102, 1985, pp. 93-117. 